\title{
Context effects on tempo and pleasantness judgments for Beatles songs
}

\author{
Matthew A. Rashotte • Douglas H. Wedell
}

Published online: 13 January 2012

(C) Psychonomic Society, Inc. 2012

\begin{abstract}
Context effects on tempo and pleasantness judgments of different tempos were demonstrated in three experiments using Beatles songs. In Experiments 1 and 2, we explored how listening to versions of the same song that were played at different tempos affected tempo and pleasantness ratings. In both experiments, contrast effects were found on judgments of tempo, with target tempos rated faster when context tempos were slow than when they were fast. In both experiments, we also showed that the peak of the pleasantness rating function shifted toward the values of the context tempos, reflecting disordinal context effects on pleasantness relationships. Familiarity with the songs did not moderate these effects, and shifts in tempo ratings did not correlate with shifts in most pleasant target tempos when context was manipulated within subjects. In Experiment 3, we examined how manipulations of context tempos for one song affected judgments of the same song as compared with judgments of other more or less similar songs. For tempo ratings, contrast effects transferred to ratings of a similar song, but for pleasantness ratings, assimilative shifts of ideals were found only for the same song and not for similar songs. This pattern of results was supportive of independent bases for the two context effects.
\end{abstract}

Keywords Music cognition - Sound recognition · Decision making · Context · Judgment · Assimilation · Contrast . Shifting Standards $\cdot$ Pleasantness $\cdot$ Tempo

\footnotetext{
M. A. Rashotte

Extended University at the University of South Carolina, Columbia, SC, USA

D. H. Wedell $(\bowtie)$

Department of Psychology, University of South Carolina, Columbia, SC 29208, USA

e-mail: wedell@sc.edu
}

The literature on context effects in judgment provides strong support for the assertion that recently experienced stimuli can shift judgments of and preferences for the currently experienced stimulus (for reviews, see Parducci, 1995; Wedell, Hicklin, \& Smarandescu, 2007), as explained in detail below. Given these pervasive effects, one would expect that altering the features of recently experienced musical clips would likewise affect feature judgments and liking for subsequent music. For instance, the tempo of a presented musical clip may be judged fast or slow, depending on the recent set of tempos one has experienced. Furthermore, the most pleasant tempo for a song may change as a function of the tempos one has recently experienced for that song. In a set of three experiments, we investigated how manipulating tempo for Beatles songs affects both judgments of tempo and tempo pleasantness. We investigated tempo because it has been demonstrated to be an important determinant of how songs are evaluated (Deutsch, 1999; LeBlanc, Colman, McCrary, Sherrill, \& Malin, 1988; LeBlanc \& McCrary, 1983), and it is a feature of the music that can be digitally altered with minimal disturbance of other musical attributes such as pitch, loudness, or timbre. In our studies, we attempted to determine whether context effects found in other domains extended to the domain of music in ways not previously demonstrated (i.e., Parker, Bascom, Rabinovitz, \& Zellner, 2008), as well as how context effects on tempo judgment relate to context effects on judged pleasantness of tempo. Before describing these experiments, we will first review the nature of the context effects we will be examining and how these may apply to judgments of tempo and pleasantness for musical clips. The present research builds on the framework described by Wedell and Pettibone (1999), who explored how the nature of context effects on judgments depends critically on the relationship of the judgment scale to the underlying stimulus attribute. Figure 1 


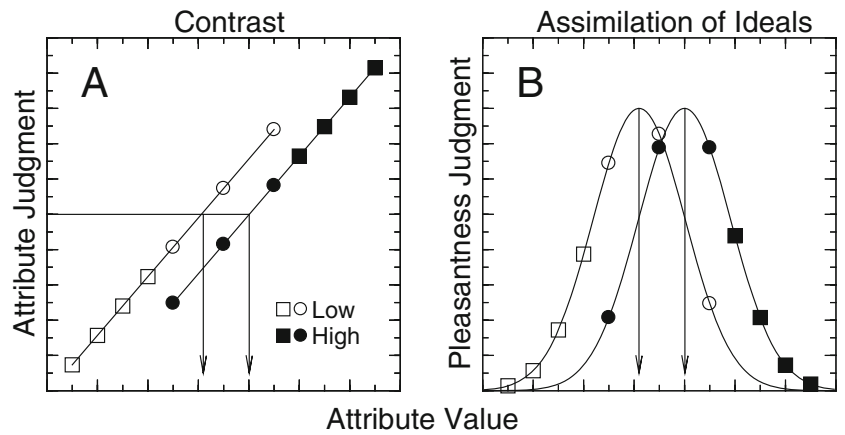

Fig. 1 Illustration of contextual contrast effects in attribute judgments (a) and assimilation of ideals for pleasantness judgments (b). Arrows in a indicate how the judgment-mediated model predicts different ideal points for low and high contexts. Arrows in b show the differences in ideal points for low and high contexts typically observed. Target values common to both contexts are shown as circles, and context values are shown as squares

illustrates the two types of relationships and the corresponding anticipated context effects. In panel A, judgments describe the magnitudes of a given attribute across various stimuli, such as judging the tempos of different pieces of music. In this case, the expected relationship between judgments and stimulus values is monotonic (or ordinal) such that faster tempos receive higher judgments than slower tempos. For ordinal relationships between judgments and attribute values, the anticipated effect of context is contrast: The same tempo should be judged faster in the context of slower tempos than in the context of faster contexts. Contextual contrast is well explained by Parducci's (1995) range-frequency theory of judgment, according to which the judged value of a stimulus is dependent on its rank in the distribution (the frequency principle) and its value relative to the minimum and maximum stimuli brought to mind at the time of judgment (the range principle).

The range-frequency judgment model can be expressed as follows:

$$
\begin{aligned}
& \mathrm{J}_{i k}=\mathrm{C}_{\mathrm{MIN}}+\left(\mathrm{C}_{\mathrm{MAX}}-\mathrm{C}_{\mathrm{MIN}}\right)\left[w\left(\mathrm{~S}_{i}-\mathrm{S}_{\mathrm{MIN}, k}\right) /\right. \\
& \left.\left(\mathrm{S}_{\mathrm{MAX}, k}-\mathrm{S}_{\mathrm{MIN}, k}\right)+(1-w)\left(\operatorname{Rank}_{i k}-1\right) /\left(\mathrm{N}_{k}-1\right)\right],
\end{aligned}
$$

where $\mathrm{J}_{i k}$ is the judgment of stimulus $i$ in context $k$ on a scale bounded by rating categories $\mathrm{C}_{\mathrm{MIN}}$ and $\mathrm{C}_{\mathrm{MAX}}, w$ is the relative weighting of the range principle, $\mathrm{S}_{i}$ is the contextindependent value of stimulus $i, \mathrm{~S}_{\mathrm{MIN}, k}$ and $\mathrm{S}_{\mathrm{MAX}, k}$ are the minimum and maximum values brought to mind that define the range of context $k, \operatorname{rank}_{i k}$ is the rank of stimulus $i$ in context $k$, with 1 and $\mathrm{N}_{k}$ representing the minimum and maximum ranks in context $k$. Range-frequency theory predicts how shifts in the distribution (via shifts in range or relative frequencies) produce shifts in judgments that reflect contrast effects (Parducci, 1995).
In panel A of Fig. 1, the low context set includes four values that are lower than the values of the three target stimuli, which are common to both distributions (targets are shown as circles, whereas squares signify contextual values). Including these lower values will extend the range downward and increase the percentile ranks of the targets, thus displacing judgments upward. Conversely, the high context will extend the range upward and decrease the percentile ranks of the targets, displacing judgments downward. Such contrast effects are found for a wide variety of stimuli and judgments (for reviews, see Parducci, 1995; Wedell et al., 2007).

Panel B of Fig. 1 presents a nonmonotonic (disordinal) relationship between judgments and attribute values, with judgments rising with increased value up to a point and then decreasing. This type of single-peaked function is typical of judgments related to pleasantness, such as attitude judgments (Eagly \& Chaiken, 1993). The attribute value at which the function peaks is referred to as an ideal point, reflecting the most pleasant attribute value (Coombs, 1964). Research exploring context effects on ideal points has primarily examined preference-related judgments in perceptual domains and has overwhelmingly found that the ideal point shifts toward the values of contextual stimuli (Hicklin \& Wedell, 2007; Pettibone \& Wedell, 2007; Riskey, Parducci, \& Beauchamp, 1979; Wedell \& Pettibone, 1999; Wedell, Santoyo \& Pettibone, 2005; although for an exception, see Cooke, Janiszewski, Cunha, Nasco, \& de Wilde, 2004). Panel B of Fig. 1 illustrates the typical context effect on ideals, with the ideal point, defined by the peak of the curve, shifting toward lower values in the low context and higher values in the high context. Note that these shifts produce a disordinal interaction in which pleasantness relations reverse for some targets. For example, in panel B of Fig. 1, the lowest target is rated higher than the highest target in the low context, but this relationship is reversed in the high context.

\section{Judgment-mediated predictions}

Although the range-frequency theory explanation of contextual contrast is well accepted, opinions about the origins of the assimilative shift of ideals are somewhat less settled. Wedell and Pettibone (1999) described two potential processes that might underlie this effect. The first they referred to as judgment mediated, according to which the contrastive shifts in the judgment of the attribute drive the assimilative shifts of ideal points. The long arrows in panel A of Fig. 1 help to illustrate this idea. In this example, the ideal is assumed to be defined by a fixed point on the attribute judgment scale. The ideal point then corresponds to different stimulus values. As illustrated in panel A, the elevated 
judgment function for the low context results in a lower ideal point than the relationally lower judgment function for the high context. According to the judgment-mediated model, the two types of context effects - contrast in judgment and assimilation of ideals in pleasantness - should show a close correspondence.

The judgment-mediated model can be expressed within a Gaussian ideal-point framework as follows:

$\mathrm{A}_{i k}=a+b \exp \left[-c\left(\mathrm{~J}_{i k}-\mathrm{J}_{\mathrm{IDEAL}}\right)^{2}\right]$,

where $\mathrm{A}_{i k}$ is the attractiveness judgment of stimulus $i$ in context $k$ on the designated rating scale, $c$ is a discriminability parameter reflecting how quickly the function rises and falls, $\mathrm{J}_{i k}$ is the contextual judgment of the stimulus from range-frequency theory, and $\mathrm{J}_{\text {IDEAL }}$ is the judgment value deemed ideal. The constants $a$ and $b$ linearly transform the attractiveness judgment from a $0-1$ scale to the rating scale. Equation 2 describes a normal curve function that peaks at the stimulus whose judged value is equal to the ideal judged value. For many domains, the ideal should fall toward the center of the judgment scale, reflecting the avoidance of extremes. Presumably for song stimuli the ideal will vary with the type of music. For example, a sad song might have a slower ideal tempo than a happy song. Consistent with this idea, research by Quin and Watt (2006) demonstrates that people have different ideal tempos for different types of songs.

One interpretation of the judgment-mediated model is that the contrastive shifts on judged tempo reflect shifts in perceived tempo, so that the tempo perceived as the ideal corresponds to an objective tempo that is shifted toward the contextual set of values. An important implication of the judgment-mediated model is that contrastive shifts in descriptive evaluations should correlate with assimilative shifts of ideals derived from pleasantness judgments. We come back to this point when discussing the prototype model of these effects.

\section{Prototype based predictions}

An alternative to the judgment-mediated interpretation is the idea that shifts in the ideal point may be driven by a process that is independent of shifts in the attribute judgments. One such candidate process proposed by Wedell and Pettibone (1999) was that ideals were in part determined by the prototypical value for the stimulus, where the prototype is often represented by the average of experienced values (Reed, 1972). Linking the ideal to the prototype is consistent with demonstrations that the average of faces is judged more attractive than the constituent faces (Langlois \& Roggman, 1990). More relevant to the domain of music, Repp (1997) demonstrated how the average of musical performances (i.e., averaging of MIDI recordings) was judged higher in quality than nearly all the constituent performances, although it was judged lower in individuality. Further support for the concept of abstracting an average is provided by the work of Ariely (2001), who demonstrated that averages can be computed quickly and used in perceptual estimation. Extraction of a running average is also consistent with early theories of context effects, such as Helson's (1964) adaptation level theory.

More generally, the literature on categorization provides ample evidence that the graded nature of categories (found in typicality judgments, inductive inference, truth verification, etc.) can be explained in terms of the distance of a stimulus from the category prototype (Homa, 1984). However, within this literature, there is a debate concerning whether prototypes are encoded in memory and updated with each new experience or whether category exemplars are encoded and retrieved at the time of judgment (Nosofsky \& Zaki, 2002). Hintzman (1986) demonstrated how exemplar models account for prototype effects by constructing a prototype from the retrieved exemplars. An advantage of exemplar models is that they can account for contextual effects that prototype models cannot, since context may be used to recruit the particular exemplars used to extract a prototype. Because shifts in ideals occur rapidly with shifts in context for both unfamiliar and familiar stimuli (Pettibone \& Wedell, 2007; Wedell \& Pettibone, 1999; Wedell et al., 2005), we posit a prototype model in which the prototype is constructed based on the retrieved exemplars, with recent exemplars weighted more heavily than older exemplars.

Within our prototype model, we represent the ideal point as an average of recent and older memories as follows:

$\mathrm{S}_{\mathrm{IDEAL}, \mathrm{k}}=z \mu_{k}+(1-z) \mu_{0}$,

where $\mathrm{S}_{\text {IDEAL,k }}$ is the stimulus value representing the ideal point in context $k, z$ is the relative weighting of the recent context $k, \mu_{k}$ is the mean of the stimulus values in context $k$, and $\mu_{0}$ is the mean of the stimulus values prior to the current context. Equation 3 provides a conceptual framework for our use of the term prototype, but several aspects of the model are left unspecified. First, it is not clear what constitutes the recent context. It could be the last few trials, or it could be a more extensive set of trials. Second, the model does not specify how frequency might affect weighting. If the prior prototype, $\mu_{0}$, is based on a higher number of instances, will the weight of the current context, $z$, be reduced? If so, one would expect familiarity with the stimuli to reduce contextual dependence, although in many domains this does not seem to be the case (O'Reilly, Leitch, \& Wedell, 2004; Perrett, 1971). We examined this issue in Experiments 1 and 2 by including a measure of song familiarity in our analyses. 
The prototype model then can be expressed as follows:

$\mathrm{A}_{i k}=a+b \exp \left[-c\left(\mathrm{~S}_{i}-\mathrm{S}_{\mathrm{IDEAL}, k}\right)^{2}\right]$

where the $\mathrm{A}_{i k}$ is the attractiveness judgment of stimulus ${ }_{i}$ in context $k, c$ is a discriminability parameter of the normal curve function, $\mathrm{S}_{i}$ is the context-invariant scale value of the stimulus, and $\mathrm{S}_{\mathrm{IDEAL}, k}$ is the stimulus value deemed ideal according to the average of recent and prior experiences as described in Eq. 3. Once again, $a$ and $b$ are scaling constants that relate the $0-1$ judgment to the rating scale being used. By comparing Eqs. 2 and 4, one can see that the judgmentmediated model is based on contextually altered stimulus values and a constant ideal, whereas the prototype model is based on contextually independent scale values and an ideal that is altered by the recent set of stimuli. Although the predictions of the two models will often be very similar, a key difference is that the judgment-mediated model entails shifts in pleasantness when attribute judgments shift, but the prototype model does not.

If attribute contrast and ideal-point assimilation derive from different processes, then it should be possible to find circumstances in which the two effects are dissociated. Although Wedell and Pettibone's (1999) research showed a moderate correlation between these effects, subsequent research has provided evidence for dissociation. For example, Wedell et al. (2005) demonstrated that participants in their sample who held highly negative self-appraisals of body image did not show the assimilation of ideals effect on body image evaluation, even though they did show the usual contrast effect on judgments of thinness. Likewise, Pettibone and Wedell (2007) demonstrated that when evaluations were prompted by labels for stimuli learned in the same encoding context, large contrast effects occurred for feature judgments with no accompanying shifts in ideals for pleasantness judgments. In two other experiments in which context was manipulated within subjects, Pettibone and Wedell showed that contextual contrast did not significantly correlate with ideal-point assimilation. Thus, there is mounting evidence for an independent basis for these two effects.

\section{Overview of experiments}

In the present article, we report three experiments exploring how these two types of context effects apply to judgments of Beatles music. Using music by The Beatles resulted in different levels of song familiarity across our sample of undergraduate participants, enabling us to address the question of how different levels of song familiarity affect contextual judgment. For instance, will high and low familiarity listeners show the same degree of contrast effects and idealpoint pleasantness shifts? If so, this would be evidence for the powerful effect of recently experienced contextual stimuli on these judgment processes. We manipulated the tempo of Beatles songs using computer software and created fast and slow tempo contexts. Embedded in these contexts were target tempo clips that included one with the original tempo of the song. The effects of contextual clips were assessed with these target clips. Prior research has shown that tempo judgments follow an ordinal relationship with tempo but that pleasantness judgments follow a single peaked relationship, with an intermediate tempo being rated most pleasant (Holbrook \& Anand, 1990; Quin \& Watt, 2006). Accordingly, we predicted that participants would show contrast effects on tempo judgments, with target tempos being judged faster in the slow tempo context than in the fast tempo context. We also predicted that participants would show assimilation of ideal points toward the mean of the recently experienced contextual clips. For example, the participants in the fast context should rate a faster target tempo as more pleasant than should participants in the slow context. However, song familiarity may moderate these contextual effects in listeners.

Our experimental designs also provided some tests of the judgment-mediated model of assimilation of ideals. In Experiments 1 and 2, we shifted context within subjects so that the magnitude of the contrast effect in tempo ratings and the assimilation of ideals effect in pleasantness ratings could be gauged for each participant. The judgment-mediated model predicts a significant correlation between these effects, given their common basis. The prototype explanation of ideal-point assimilation does not. In Experiment 3, we also provided a test of these two models by examining the transfer of context effects across different target songs. Typical models of contrast predict effects that generalize to similar stimuli but not to dissimilar stimuli (Brown, 1953; Zellner, Rohm, Bassetti, \& Parker, 2003), so the judgmentmediated model would predict transfer to songs high in similarity for both tempo and tempo pleasantness judgments. A prototype explanation of assimilation of ideals would be expected to be more song specific, since ideal point comparisons would likely be based on recruitment of various instances of the same song. Thus, although contrast may extend to similar songs, assimilation of ideals may be song specific according to the prototype view.

\section{Experiment 1}

Experiment 1 was conducted to test whether manipulating tempos of a Beatles song, Sergeant Pepper's Lonely Hearts Club Band (SP), would produce the two basic effects of context described in Fig. 1: contrast for tempo judgments and assimilation of ideals for tempo pleasantness judgments. Figure 2 presents the basic design of the experiment, which 
Fig. 2 Flow diagram describing procedural details of Experiments 1 and 2
Experiments 1 and 2

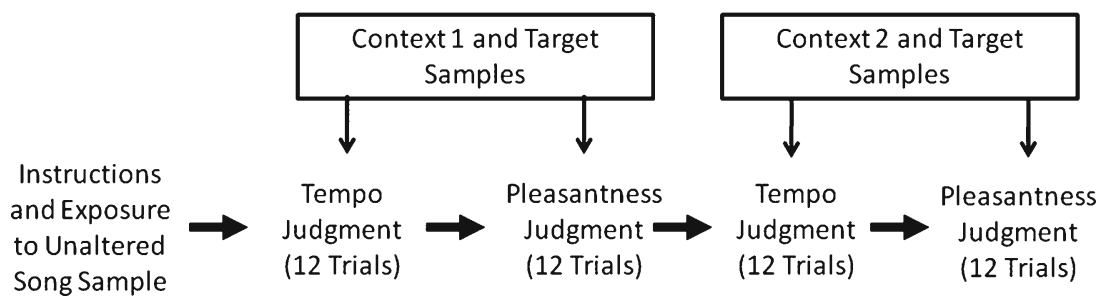

consisted of both between-subjects and within-subjects manipulations of context. The first two sets of ratings provide a between-subjects test of context effects and a gauge of the magnitude of these effects when only a single context has been presented. By reversing the contextual distribution for sets 3 and 4, we are able to examine how much contextual judgment changes despite expected carry-over effects from the first context

Evaluating each participant in each context provides within-subjects measures of contrast on tempo judgments and assimilation of ideals on pleasantness judgments that can be used to test predictions of the judgment-mediated model. One way to interpret the judgment-mediated model is that changes in the tempo judgments reflect changes in the perceived tempo of the songs and not just mapping of stimulus values to response values. If this is the case, and the ideal preferred tempo remains constant, then contrast on tempo perception should produce assimilation of ideals in pleasantness ratings, as described by Eq. 2. Thus, the judgment-mediated model predicts that there should be a positive correlation between indices of contrast and assimilation of ideals calculated for each participant. Although previous research using a similar design has not found a significant positive correlation between these indices (Pettibone \& Wedell, 2007), this proposition has not been tested with music stimuli.

Indeed there is evidence that perceived tempo may be sensitive to tempo context. Research using comparative judgments of short tone sequences has revealed that tempo perception adapts to global and local tempo contexts (Jones \& McAuley, 2005; McAuley \& Miller, 2007). For instance, the point of subjective equality for the same $500-\mathrm{ms}$ tone sequences was inferred to be slower in a slow global context but faster in a fast global context. Although it is difficult to relate results from comparative to absolute judgments, this result provides support for the possibility of a perceptionmediated effect. Alternatively, the work of McAuley and colleagues implies that tempo perception is mediated by a tendency to form and use a prototypical stimulus value based on the recent set of contextual values. This interpretation is consistent with the prototype model of pleasantness judgment we describe.
At the end of the four sets of ratings, participants in Experiment 1 rated their familiarity with the song $(S P)$. Familiarity ratings provide a way to assess the degree to which previous exposure to the song might moderate context effects. Extant research suggests that tempo is stored with a high degree of accuracy for songs with which people are very familiar. Levitin and Cook (1996) asked people to sing songs they knew well and found that the majority came within $8 \%$ of the actual tempo. This result implies that it might be difficult to shift tempo pleasantness away from the original tempo for participants who are highly familiar with the song in our experiment. Moreover, research indicates that listeners can identify the original recording of a song from a tempo-altered version of a different performance of that song digitally altered to have the same tempo (Honing, 2006). Honing and Ladinig (2009) also found that identification of the original unaltered-tempo version increased with exposure to the musical idiom from which the songs were drawn. Thus, participants in our study who were highly familiar with the song may have been better able to distinguish the original from the altered tempo versions. These findings, taken together, suggest that familiarity might moderate context effects on tempo and pleasantness judgments in our studies, with reduced contextual effects expected for those who are most familiar with the music. However, none of these studies manipulated context; thus, it is unclear to what degree familiarity will be a moderator. Indeed, research in other domains of judgment suggests that exposure to and expertise with the stimulus domain only slightly reduces context effects of this kind (O'Reilly et al., 2004; Perrett, 1971).

\section{Method}

Participants and design Participants were 83 undergraduates from the University of South Carolina who received course credit for volunteering. The experimental design was based on two different testing blocks. In the first block, participants were randomly assigned to either the fast or slow tempo context, so tempo context was manipulated between subjects. The slow tempo context consisted of seven clips with tempos of $-30 \%,-27 \%,-24 \%,-21 \%$, 
$-18 \%,-15 \%$, and $-9 \%$ of the original. The fast tempo context consisted of seven clips with tempos of $30 \%, 27 \%, 24 \%$, $21 \%, 18 \%, 15 \%$, and $9 \%$. Additionally, both sets included five target clips with tempos of $-12 \%,-6 \%, 0 \%, 6 \%$, and $12 \%$. The original tempo (i.e., $0 \%$ ) thus was included as a target. The resulting beats per minute (bpm) for each clip is shown in Table 1. Nine-point rating scales were used to make tempo and pleasantness ratings. As shown in Fig. 2, following the first block of testing, participants repeated the task with the other contextual set of song clips. This design allowed for assessment of contextual shifts within subjects, as well as of how changes in contrast scores across contexts related to changes in assimilation of ideals across contexts. As shown in Fig. 2, ratings of tempo (sets 1 and 3) always preceded ratings of tempo pleasantness (sets 2 and 4). Previous research has demonstrated that manipulating the order of these two tasks does not affect the magnitude of the context effects obtained (Pettibone \& Wedell, 2007; Wedell \& Pettibone, 1999; Wedell et al., 2005).

Materials and apparatus All experimental materials were presented on desktop computers with 17-in. monitors and audio headphones that covered the ear and provided a highquality listening experience. We utilized the recently released digitally remastered Beatles compact discs from 2009. Tracks were copied to a computer using AVS audio converter 6.1 as WAV files. Bit rate was set at 1,411 kps,

Table 1 Beats per minute for contextual and target songs

\begin{tabular}{lllllll}
\hline Song Type & \% Change & $S P$ & $P B W$ & SLU & LWR & SOM \\
\hline Context & -30 & 67 & 109 & & & 46 \\
Context & -27 & 69 & 114 & & & 48 \\
Context & -24 & 72 & 119 & & & 50 \\
Context & -21 & 75 & 123 & & & 52 \\
Context & -18 & 78 & 128 & & & 54 \\
Context & -15 & 81 & 133 & & & 56 \\
Target & -12 & 84 & 137 & 139 & 58 & 58 \\
Target & -6 & 89 & 147 & 149 & 62 & 62 \\
Target & 0 & 95 & 156 & 158 & 66 & 66 \\
Target & 6 & 100 & 165 & 167 & 70 & 70 \\
Target & 12 & 106 & 175 & 177 & 74 & 74 \\
Context & 15 & 109 & 179 & & & 76 \\
Context & 18 & 112 & 184 & & & 78 \\
Context & 21 & 115 & 189 & & & 80 \\
Context & 24 & 118 & 193 & & & 82 \\
Context & 27 & 121 & 198 & & & 84 \\
Context & 30 & 124 & 203 & & & 86 \\
\hline
\end{tabular}

$\%$ Change is the percent the original tempo was speeded up or slowed down. SP Sergeant Pepper's Lonely Hearts Club Band; PBW Paperback Writer; SLU She Loves You; LWR The Long and Winding Road; SOM = Something sample rate was $44,100 \mathrm{~Hz}$, and sample size was 16 bit. The WAV files were then opened in Audacity 1.2.6 and edited. A $14.47 \mathrm{~s}$ section from the song $S P$ served as the clip. The clip was taken from approximately $0: 20$ to $0: 34$ in the song and included the lyrics, "It was twenty years ago today, Sergeant Pepper told the band to play, they've been going in and out of style, but they're guaranteed to raise a smile, so may I introduce to you." The Beatles set the original tempo of this song at $95 \mathrm{bpm}$ (Leonard, 1995). Using this clip, we created 18 tempo-altered clips with Audacity while holding constant other features of the song such as pitch and loudness. The clip was tempo transformed in Audacity using the "Change tempo without changing pitch" function. Each file was transformed by a percent change amount and was then exported as WAV files. The tempo manipulations systematically affected the length of the clip. When the speed of the original clip was increased by $30 \%$, the clip's duration was $11.13 \mathrm{~s}$, and when the speed was decreased by $30 \%$, the clip's duration was $20.65 \mathrm{~s}$. E-prime was used to administer the experiment and to collect tempo and pleasantness ratings.

Procedure Participants were tested in groups of up to four in a large laboratory room with computers spaced about $2 \mathrm{~m}$ apart. Figure 2 shows how participants completed each step of the experiment. First, instructions informed participants that they would hear clips from $S P$ by the Beatles played at different tempos. After each clip, participants would rate it in terms of tempo or pleasantness. Before any ratings were made, participants listened to the original tempo clip (i.e., $0 \%$ ). Specifically, they were instructed to click on the mouse to hear the original unaltered portion of the song. Each block of the experiment was divided into two phases. In the first phase, participants rated each clip in terms of tempo on a 9point scale anchored by $1=$ Slow tempo and $9=$ Fast tempo . During this phase, a total of 12 clips (five target and seven contextual clips) were presented to participants in random order. In the second phase, participants again heard the 12 clips in random order but rated each clip in terms of pleasantness on a 9-point scale anchored by $1=$ not at all pleasant tempo to $9=$ very pleasant tempo. After participants completed their first block of testing, they began the second testing block. This second block was identical to the first except that participants were assigned the opposite level of context. After completing the second block, participants gave a familiarity rating for $S P$ on a 9-point scale anchored by $1=$ not at all familiar to $9=$ very very familiar .

Results

Results for between-subjects and within-subjects comparisons are reported in separate sections below. For each 
design, repeated measures ANOVAs were conducted on tempo and pleasantness ratings of the targets, with partial $\eta^{2}$ provided as a measure of effect size for significant effects. To aid in the theoretical interpretation of the results, we conducted model fits to the group mean tempo ratings and pleasantness ratings. Rather than engage in model testing of different parameterizations of the models, we estimated a simple version of each model that fit the data well and provided interpretable parameter values shown in Table 2. Additionally, we compared fitted ideal points with those predicted by the judgment-mediated model, as shown in Table 3. These modeling results are described in three separate sections. A final section of the Results is devoted to analyses of individual differences.

Between-subjects comparisons Participants were assigned to either the fast or the slow tempo context for their first two sets of ratings so that comparisons between context are also between-subjects comparisons for these data. Figure 3 presents the mean ratings for tempo (panel A) and pleasantness (panel B), along with the model fits. Both panels show large context effects consistent with predictions.

A 2 (context) $\times 5$ (target) repeated measures ANOVA was conducted on tempo ratings of the five target tempos. The significant main effect of context, $F(1,81)=95.5$, partial $\eta^{2}=0.541, p<.001$, reflected the predicted contrast effect in which the same tempo was rated faster in the slow context than in the fast context. The significant main effect of target, $F(4,324)=108.1$, partial $\eta^{2}=0.572, p<.001$, simply reflected the fact that tempo ratings were highly sensitive to the actual differences in tempo between targets. The lack of a Context $\times$ Target interaction, $F(4,324)=0.4, p>.05$, indicated that the context effect did not significantly differ across targets.

A 2 (context) $\times 5$ (target) repeated measures ANOVA was also conducted on pleasantness ratings of the five target tempos. The significant main effect of context, $F$ $(1,81)=33.4$, partial $\eta^{2}=0.292, p<.001$, reflected higher pleasantness ratings for the target tempos in the slow context. The significant main effect of target, $F(4$, $324)=23.0$, partial $\eta^{2}=0.221, p<.001$, reflected the predicted curvilinear relation between pleasantness and tempo for the target values. The significant Context $\times$ Target interaction, $F(4,324)=11.7$, partial $\eta^{2}=0.126, p<$ .001 , was consistent with the predicted crossover interaction in which the peak of the pleasantness rating function was shifted to a faster target tempo in the fast context than in the slow context. This interpretation is supported by the statistically significant linear component of the interaction, $F(1,81)=39.6$, partial $\eta^{2}=0.328, p<.001$.

Although the most accurate way to assess ideal points for each participant is to fit Eq. 4 to each (see Wedell \& Pettibone, 1999, for an example), this method is problematic in significance testing for two reasons. First, when the function is monotonic, the fitted value of the ideal point estimate is very unstable and may be extreme in magnitude. Second, because contextual values are asymmetric across conditions, artifacts may be introduced into the estimation process by the inclusion of different contextual values across conditions. A measure that does not suffer these
Table 2 Parameter values for range-frequency model and Gaussian ideal-point model fits

For range-frequency fits to tempo ratings,

$\mathrm{S}_{\mathrm{MIN}}=$ minimum value defining the range, $\mathrm{S}_{\mathrm{MAX}}=$ maximum value defining the range; for Gaussian ideal-point fits to pleasantness ratings, $\mathrm{b}=$ maximum height of function, $\mathrm{c}=$ narrowness of function, Ideal $=$ location of peak of function

\begin{tabular}{|c|c|c|c|c|c|c|c|}
\hline Experiment & Condition & Parameter & Slow & Fast & Parameter & Slow & Fast \\
\hline \multirow[t]{3}{*}{1} & \multirow[t]{3}{*}{ Slow to fast } & $\mathrm{S}_{\mathrm{MIN}}$ & -34.74 & -35.11 & $\mathrm{~b}$ & 5.96 & 5.54 \\
\hline & & $\mathrm{S}_{\mathrm{MAX}}$ & 23.94 & 32.65 & $\mathrm{c}$ & 0.0018 & 0.0012 \\
\hline & & & & & Ideal & 2.10 & 12.23 \\
\hline \multirow[t]{3}{*}{1} & \multirow[t]{3}{*}{ Fast to Slow } & $\mathrm{S}_{\mathrm{MIN}}$ & -33.97 & -22.72 & $\mathrm{~b}$ & 5.45 & 5.29 \\
\hline & & $\mathrm{S}_{\mathrm{MAX}}$ & 39.45 & 38.58 & $\mathrm{c}$ & 0.0010 & 0.0011 \\
\hline & & & & & Ideal & 9.73 & 16.83 \\
\hline \multirow[t]{3}{*}{2} & \multirow[t]{3}{*}{ Slow to fast } & $\mathrm{S}_{\mathrm{MIN}}$ & -39.31 & -39.30 & $\mathrm{~b}$ & 5.88 & 5.32 \\
\hline & & $\mathrm{S}_{\mathrm{MAX}}$ & 20.44 & 32.45 & $\mathrm{c}$ & 0.0017 & 0.0010 \\
\hline & & & & & Ideal & -1.50 & 6.94 \\
\hline \multirow[t]{3}{*}{2} & \multirow[t]{3}{*}{ Fast to Slow } & $\mathrm{S}_{\mathrm{MIN}}$ & -34.83 & -25.94 & $\mathrm{~b}$ & 6.11 & 5.72 \\
\hline & & $\mathrm{S}_{\mathrm{MAX}}$ & 30.66 & 36.08 & $\mathrm{c}$ & 0.0016 & 0.0016 \\
\hline & & & & & Ideal & 1.72 & 12.22 \\
\hline \multirow[t]{3}{*}{3} & \multirow[t]{3}{*}{$P B W$} & $\mathrm{~S}_{\mathrm{MIN}}$ & -52.56 & -38.92 & $\mathrm{~b}$ & 5.96 & 5.06 \\
\hline & & $\mathrm{S}_{\mathrm{MAX}}$ & 17.06 & 44.61 & $\mathrm{c}$ & 0.0015 & 0.0012 \\
\hline & & & & & Ideal & -5.72 & 16.43 \\
\hline \multirow[t]{3}{*}{3} & \multirow[t]{3}{*}{$S O M$} & $\mathrm{~S}_{\mathrm{MIN}}$ & -42.88 & -18.63 & $\mathrm{~b}$ & 4.88 & 4.71 \\
\hline & & $\mathrm{S}_{\mathrm{MAX}}$ & 64.40 & 88.96 & $\mathrm{c}$ & 0.0008 & 0.0009 \\
\hline & & & & & Ideal & 4.85 & 24.13 \\
\hline
\end{tabular}


Table 3 Comparison of judgment-mediated model-predicted ideals with inferred ideals (Experiments 1 and 2)

\begin{tabular}{|c|c|c|c|c|c|c|c|c|}
\hline Experiment & Song & Context & Condition & $\mathrm{S}_{\mathrm{MIN}}$ & $\mathrm{S}_{\mathrm{MAX}}$ & $\mathrm{J}_{\text {Ideal }}$ & Predicted Ideal & Inferred Ideal \\
\hline 1 & $S P$ & slow & $s-f$ & -34.74 & 23.94 & 6.14 & 2.92 & 2.1 \\
\hline 1 & $S P$ & fast & $s-f$ & -35.11 & 32.65 & 6.14 & 8.37 & 12.23 \\
\hline 1 & $S P$ & slow & $\mathrm{f}-\mathrm{s}$ & -33.97 & 39.45 & 6.14 & 13.14 & 9.73 \\
\hline 1 & $S P$ & fast & $\mathrm{f}-\mathrm{s}$ & -22.72 & 38.58 & 6.14 & 16.62 & 16.83 \\
\hline 2 & $P B W$ & slow & $s-f$ & -39.31 & 20.44 & 5.90 & -2.7 & -1.5 \\
\hline 2 & $P B W$ & fast & $s-f$ & -39.3 & 32.45 & 5.90 & 4.66 & 6.94 \\
\hline 2 & $P B W$ & slow & f-s & -34.83 & 30.66 & 5.90 & 5.29 & 1.72 \\
\hline 2 & $P B W$ & fast & $\mathrm{f}-\mathrm{s}$ & -25.94 & 36.08 & 5.90 & 12.06 & 12.22 \\
\hline
\end{tabular}

SP Sergeant Pepper's Lonely Hearts Club Band; PBW Paperback Writer; $s-f$ slow then fast order; $f-s=$ fast then slow order; $S_{M I N}$ minimum subjective value from range-frequency fit, $S_{M A X}$ maximum subjective value from range-frequency fit; $J_{I D E A L}$ judgment value corresponding to the ideal in Eq. 2 Predicted ideal $=\mathrm{S}_{\mathrm{MIN}}+\left(\mathrm{J}_{\mathrm{Ideal}}-1\right)\left(\mathrm{S}_{\mathrm{MAX}}-\mathrm{S}_{\mathrm{MIN}}\right) / 8$; inferred ideal from the fit of Eq. 4

problems is the most pleasant target tempo, which is simply the target tempo that is rated highest in pleasantness. If more than one target tempo shares the highest rating, then these tempo values are averaged together. Because only target tempos shared across conditions are used, the calculated value cannot reflect artifacts resulting from including different contextual values in estimation. Because this measure is restricted to be in the range of the target values $(-12 \%$ to $12 \%$ ), it will not produce extreme and unreasonable values associated with monotonic functions. However, for this same reason, it will not be as extreme as the true ideal point value when that value lies outside the target range. We conducted analyses on the most pleasant target tempos computed as a way to more directly infer changes in ideal points. A $t$ test computed on this measure was significant, $t$ $(81)=3.02, p<.01$, with the most pleasant target tempo faster in the fast context $(M=7.28)$ than in the slow context $(M=2.37)$.

To determine the effects of familiarity, we dichotomized the familiarity ratings and included this grouping factor in the analyses described earlier (six cases were excluded from the analysis because of missing familiarity rating data for these participants). Familiarity with $S P$ varied widely across listeners $(M=4.16$ and $S D=2.91)$. Participants were classified into the high familiarity group if their familiarity rating was 5 or greater $(N=35)$ and into the low familiarity group if their rating was less than $5(N=42)$. For neither the ANOVA on tempo judgments or on pleasantness judgments did familiarity interact with context. Thus, there was no evidence that context effects were moderated by familiarity with the song. Familiarity grouping did significantly interact with target values for the ratings of pleasantness, $F(4,292)=4.09$, partial $\eta^{2}=0.053, p<.01$. Examination of the means indicated that the high familiarity group rated the faster tempos more pleasant than the original tempo, but the low familiarity group did not. Thus, the interaction did not reflect any tendency for familiarity to increase tempo pleasantness for the original tempo version of the song.

Within-subjects comparisons After rating tempo and pleasantness in one context, participants were exposed to the opposite context and rated tempo and pleasantness again. Comparing first and second block ratings then provides a within-subjects evaluation of context effects. Figure 4
Fig. 3 Mean ratings of tempo (a) and pleasantness (b) for target tempos (circles) and context tempos (squares) in slow- and fast-tempo contexts in Experiment 1 using the Beatles song Sergeant Pepper's Lonely Hearts Club Band. Results demonstrate contrast effects on tempo ratings and assimilation of ideals for pleasantness ratings. Rangefrequency fits shown for tempo ratings and Gaussian ideal point fits shown for pleasantness ratings
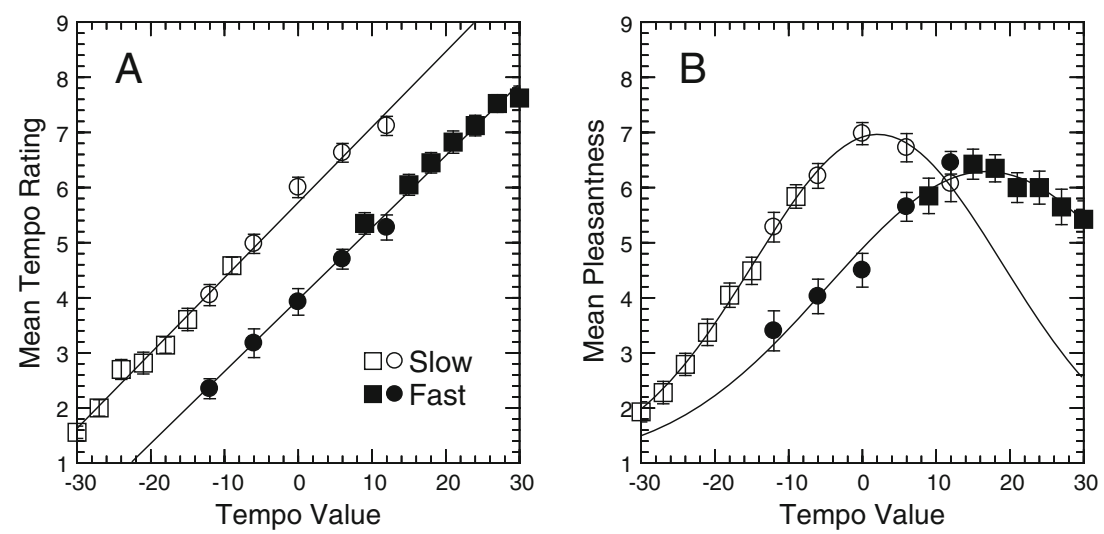
Fig. 4 Mean ratings of tempo (left panels) and pleasantness (right panels) for target tempos (circles) and context tempos (squares) in slow- and fasttempo contexts in Experiment 1 as a function of context order. Ratings in each panel are from the same participants reflecting within-subjects context effects. Range-frequency fits are shown for tempo ratings and Gaussian ideal point fits are shown for pleasantness ratings
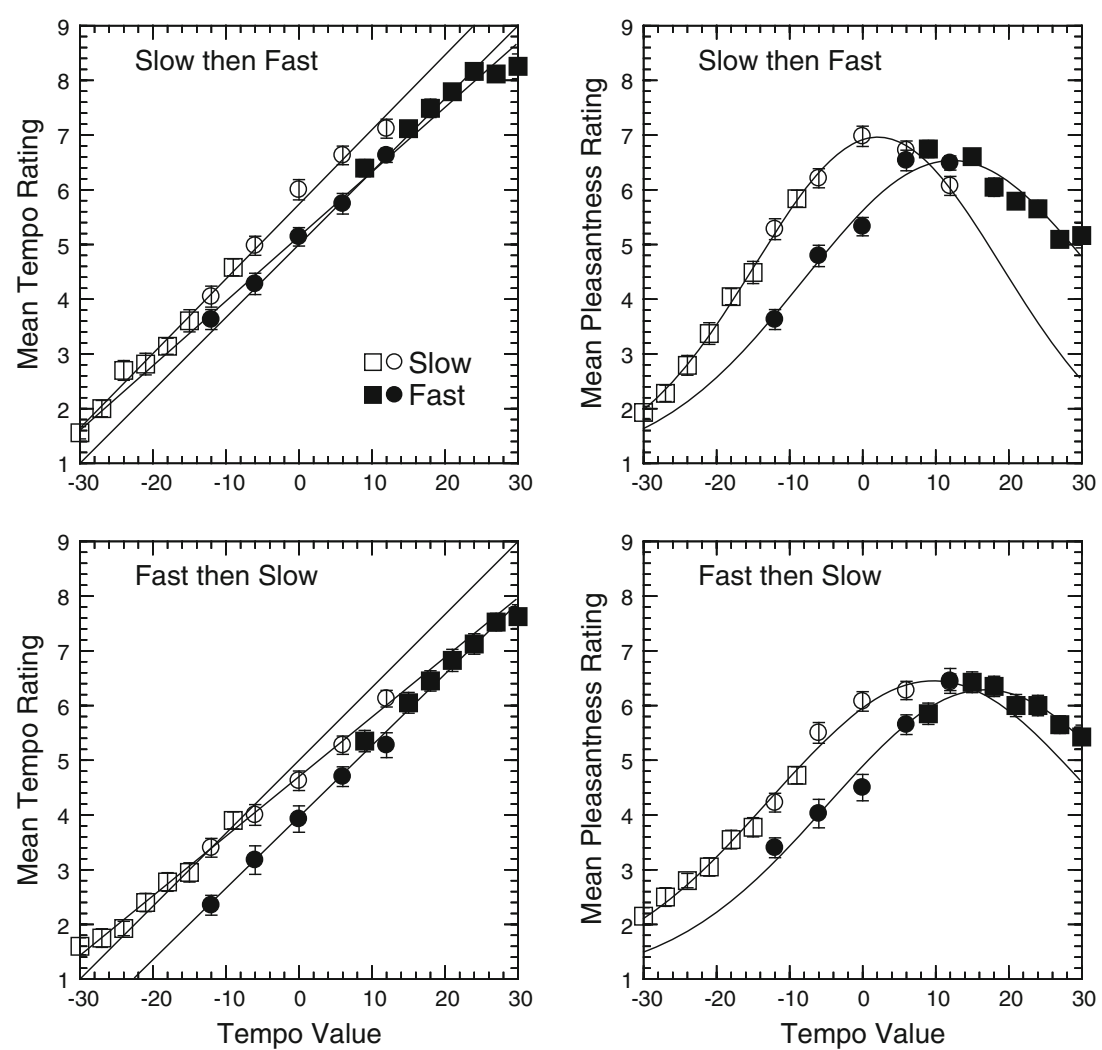

presents the data for these comparisons, along with the model fits. Each panel presents the mean ratings from the same participants in the two different contexts. As seen in the left panels, strong contrast effects were found for these within-subjects comparisons, but clear carryover effects occurred as well. The magnitude of contrast was about half that found in the between-subjects manipulation. Comparing the tempo rating functions to the diagonal, one can see that the initial context produced ratings far away from the diagonal, but these ratings shifted only about halfway back when the opposite context was subsequently presented. The pleasantness ratings show a clear difference in the peak of the functions for the slow then for the fast condition, but less of a difference for the fast then for the slow condition. Consistent with the asymmetry of shifting found in the between-subjects comparison, it was more difficult to move the ideal point to slower target tempos after the fast context established faster target tempos as more pleasant.

Parallel ANOVAs were conducted on ratings across the two blocks so that context was a within-subjects factor. A 2 (order of context) $\times 2$ (context) $\times 5$ (target) repeated measures ANOVA conducted on tempo ratings of the target tempos revealed a large effect of context, $F(1,81)=64.3$, partial $\eta^{2}=0.443, p<.001$, reflecting a strong contrast effect on tempo ratings when context was manipulated within subjects. Although this effect was quite large (a mean difference of 0.73), it was less than half the size found in the between-subjects manipulation of context (a mean difference of 1.87), reflecting carryover effects of context from the first block. Further evidence of carryover effects was found in a significant main effect of order of context, $F(1$, $81)=59.3$, partial $\eta^{2}=0.423, p<.001$, with target ratings higher when the slow context occurred first than when the fast context occurred first. The only other significant effect was a main effect of target, $F(4,324)=218.4$, partial $\eta^{2}=$ $0.729, p<.001$.

A 2 (order of context) $\times 2$ (context) $\times 5$ (target) repeated measures ANOVA conducted on pleasantness ratings of the target tempos revealed a large Context $\times$ Target interaction, $F(4,324)=13.7$, partial $\eta^{2}=0.147, p<.001$, reflecting the crossover interaction predicted by a shift in ideals toward contextual values. As predicted, the linear component of this interaction was significant, $F(1,81)=19.0$, partial $\eta^{2}=$ $0.190, p<.001$. A significant main effect of order of context, $F(1,81)=7.5$, partial $\eta^{2}=0.085, p<.01$, reflected higher pleasantness ratings when the slow context was presented first. This is consistent with asymmetric shifts of ideals so that once the fast context established slower targets as unpleasant, they did not tend to be rated as pleasant with a shift to the slower context. Evidence for carryover effects comes from a significant Order $\times$ Target interaction, $F(4$, $324)=2.9$, partial $\eta^{2}=0.035, p<.05$. If the first context 
continued to influence the ideal when the second context was presented, then the differences in ideals associated with context should have depended on which context occurred first. Accordingly, the peak of the functions averaged across contexts was at a higher tempo value when the fast context occurred first rather than second. Additional significant effects included a main effect of context, $F(1,81)=43.0$, partial $\eta^{2}=0.347, p<.001$, reflecting higher overall pleasantness ratings in the slow context, and a main effect of target, $F(4,324)=61.2$, partial $\eta^{2}=0.431, p<.001$, reflecting the predicted single peaked shape of the pleasantness rating function. No other effects were statistically significant.

Although the three-way Order $\times$ Context $\times$ Target interaction was not significant for pleasantness ratings, there appeared to be an asymmetry in the shifting of pleasantness. Those starting out in the fast context did not shift their most pleasant target tempo downward much when encountering the slow context, but those starting in the slow context did shift their most pleasant target tempo upward to a greater extent when encountering the fast context. A $t$ test comparing most pleasant target tempo when the slow context occurred first showed that these differed with context, $t(42)=2.49, p<.05$, $M_{\text {Slow }}=2.37$ and $M_{\text {Fast }}=5.86$. However, this difference was not significant when the fast context occurred first, $t(39)=$ $1.35, p>.05, M_{\text {Slow }}=5.63$ and $M_{\text {Fast }}=7.28$.

Range-frequency model fits The range-frequency model of Eq. 1 was fit to the group mean tempo ratings for the 2 (context) $\times 2$ (context order) conditions shown in the left panels of Figs. 3 and 4. Because range and frequency values were highly correlated in this design, we simplified Eq. 1 by setting $w=1.0$ and thus fit two parameters to each context, $\mathrm{S}_{\mathrm{MIN}, k}$ and $\mathrm{S}_{\mathrm{MAX}, k}$, corresponding to the minimum and maximum values defining the subjective range in each context. This version of the model was fit using least-squares linear regression. Note that $\mathrm{S}_{\mathrm{MIN}, k}$ and $\mathrm{S}_{\mathrm{MAX}, k}$ are subjective values that we infer from the data on the basis of a scalematching assumption (Parducci, 1995). Rather than fit an additive and multiplicative constant to transform internal judgments to a response scale, the scale-matching assumption posits that when the a stimulus corresponds to $\mathrm{S}_{\mathrm{MIN}, k}$ it will be assigned the lowest rating category, $\mathrm{C}_{\mathrm{MIN}}$, and that when it corresponds to $\mathrm{S}_{\mathrm{MAX}, k}$, it will be assigned the highest rating category, $\mathrm{C}_{\mathrm{MIN}}+\left(\mathrm{C}_{\mathrm{MAX}}-\mathrm{C}_{\mathrm{MIN}}\right)$. This scalematching assumption is reflected in the parameterization of Eq. 1 and is used in a similar fashion when we describe predictions from the judgment-mediated model.

Model fits are shown in the left panels of Figs. 3 and 4 as fitted lines, with a close correspondence between fitted and empirical values $\left(R^{2}=.993\right.$ for fits to the slow to fast condition, and $R^{2}=.996$ for fits to the fast to slow condition). Parameter values estimated from the range-frequency model are shown in Table 2 and help interpret the effects of order reported earlier. When the slow context is judged first, a low minimum value defining the range is established that does not change much when the fast context is subsequently judged. When the fast context is judged first, a high maximum value defining the range is established that does not change much when the slow context is subsequently judged. Thus, the range-frequency model explains the carryover effects as resulting from the maintenance of an extreme range anchor that was established in the first context when judging targets from the second context. ${ }^{1}$

Prototype model fits The Gaussian ideal-point model of Eq. 4 was fit to the group mean pleasantness ratings for the 2 (context) $\times 2$ (context order) conditions shown in the right panels of Figs. 3 and 4. In fitting the Gaussian idealpoint model of Eq. 4 , the additive constant $(a)$ was set equal to 1 , the lowest value on the rating scale, and three parameters were fit to each context. The value of $b$ reflects the height of the pleasantness rating function, the value of $c$ reflects the narrowness of the function, and the value of ideal estimates the tempo value corresponding to the peak of the function. The model was fit using iterative nonlinear regression with a least squares error function.

Model fits are shown in right panels of Figs. 3 and 4 as lines, with a close correspondence between fitted and empirical values $\left(R^{2}=.987\right.$ for fits to the slow to fast condition, and $R^{2}=.988$ for fits to the fast to slow condition). Parameter values estimated from the Gaussian ideal point model are shown in Table 2. The ideal point values reported in Table 2 can be compared to the mean most pleasant target tempos reported earlier. As expected, the differences in inferred ideal points with context are generally larger than those reported for mean most pleasant target tempo. This is largely due to the restriction on the range of tempo values possible for the latter measure (between $-12 \%$ and $12 \%$, the slowest and fastest target values). As an example, note that the inferred ideal was 16.83 for the fast context (when judged first), which is beyond the target range.

Judgment-mediated model predictions The judgmentmediated model predicts the ideal point location according

\footnotetext{
${ }^{1}$ One reviewer of this manuscript suggested that the contrast effects on tempo ratings might be due to sequential contrast in which a stimulus is judged higher on the scale when immediately preceded by a low value than a high value. We conducted a sequential analysis of the data and found that there was a significant first-order sequence effect, but this was in the assimilative rather than the contrastive direction: A tempo was judged slower when immediately preceded by a slower tempo. This pattern of effects has been reported elsewhere (Lockhead \& King, 1983) and is consistent with Parducci's (1995) assertion that the distributional effects modeled by range-frequency theory are not explained by local sequence effects.
} 
to judged tempo values and an ideal judgment value. We examined this relationship more thoroughly by comparing predictions from the judgment-mediated model of Eq. 2 to ideal points inferred by fitting Eq. 4 to the data. Table 3 shows this comparison. According to Eq. 2, one free parameter, the ideal judgment value $\left(\mathrm{J}_{\text {Ideal }}\right)$, determines the location of the ideal point once judged tempo values $\left(\mathrm{J}_{\mathrm{ik}}\right)$ are modeled. As described earlier, we successfully modeled these judged values using Eq. 1 with just two free parameters determining judgments in each context, $\mathrm{S}_{\mathrm{MIN}, k}$ and $\mathrm{S}_{\mathrm{MAX}, k}$. Thus, based on these values, each context provides an estimate of $\mathrm{J}_{\text {Ideal }}$. We averaged these estimates and used the single estimated value to predict ideal points as shown in Table 3. As can be seen, the predictions of the ideals are quite accurate for the first context encountered (i.e., the slow context in the slow to fast condition and the fast context in the fast to slow condition). However, the predictions for the ideal points for the second context encountered are not very accurate.

Individual differences analyses The significant withinsubjects effects of context enabled us to test the hypothesis that differences in judged tempo mediate the differences in the most pleasant target tempo at the individual level. If this were the case, then one would predict a significant positive correlation between the amount of contrast a participant shows in rating tempo across contexts and the amount of assimilation of ideals the participant shows in rating pleasantness across contexts. Contrast was computed for each participant by subtracting the mean of target ratings in the fast context from the mean of target ratings in the slow context. Assimilation of ideals was estimated for each participant by subtracting the most pleasant target tempo in the slow context from the most pleasant target tempo in the fast context. Inconsistent with the hypothesis of judgmentmediated effects on pleasantness, the correlation of these two context scores was close to zero, $r=.02$.

A potential problem with the use of a simple correlation to assess the correspondence between context effects on tempo judgments and pleasantness judgments is that the proposed relationship is dependent on assumptions about the tempo judgment functions. As shown in Fig. 1, the shift in ideal points predicted from panel A is based on parallel functions in low and high contexts. If the slopes of the functions change significantly across contexts, then the predicted shift in ideals can change as well. Furthermore, when comparing across participants, the steepness of the slopes of these functions should affect predicted differences in ideal points. Steeper functions will typically result in smaller shifts in ideals than flatter functions, all else being held constant. To assess these effects, we calculated for each participant the average slope across the two contexts and the difference in slopes across the two contexts based on the target tempo ratings. In a regression analysis predicting change in the most pleasant target tempo from difference in mean tempo ratings, we included these terms along with their interaction with difference in mean tempo ratings. None of the included terms significantly incremented $R^{2}$, and the model $R^{2}$ did not rise above .006, implying that the lack of correlation between these two measures was not moderated by individual slope differences or averages.

Given the single peaked nature of the pleasantness functions, one may ask to what degree are individual participants well characterized by the fits to the group mean. Assuming that all individuals follow a single peaked function, the correlation between an individual's rating function and the group function will decrease as the difference in peaks increases, and in extreme cases, it can be close to -1.0. To evaluate the correspondence between individual and average rating, we computed the correlation between each participant's rating function and the mean rating function within each order $\times$ context condition. Figure 5 presents the cumulative proportions for these correlations in each of the four conditions. Two important observations can be derived from this figure. First, there is a high degree of agreement between individual and averaged data for the first distribution encountered. For the slow-fast order, the median correlation was .844 for the slow context, with $88 \%$ having $r>$ .60. Similarly, for the fast-slow order, the median correlation was .895 for the fast context, with $90 \%$ having $r>.60$. However, the agreement of individual rating functions to the average rating function diminishes for the second set judged, presumably reflecting individual differences in the tendency to shift ideals with context. For the slow-fast order, the median correlation was .593 for the fast context, with $45 \%$ having $r>.60$. For the fast-slow order the median correlation was .667 for the slow context, with $63 \%$ having $r>.60$

\section{Discussion}

In Experiment 1, we clearly demonstrated evidence for two types of context effects operating on judgments of Beatles music: contrast for tempo ratings and assimilation of ideals for pleasantness ratings. Contrast effects were consistent with predictions of range-frequency theory (Parducci, 1995) and may be attributed to either shifts in ranks or ranges across context. Because ranks and stimulus values were highly correlated, we fit a simplified version of the range-frequency model that depended only on range values by setting $w=1$ in Eq. 1 . This model fit the mean ratings well, indicating that the shift in ratings is consistent with the two contexts bringing to mind different ranges of contextual values at the time of judgment. The range-frequency modeling also provided a simple explanation of the observed carryover effects on ratings of tempo. When adjusting to a 
Fig. 5 Cumulative proportion functions for the correlation of individual pleasantness ratings with the corresponding group average ratings as a function of order and context

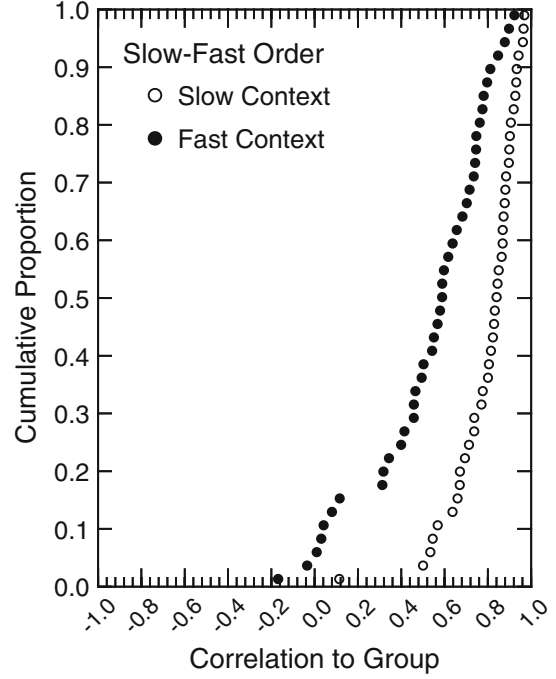

new context, the range anchor extends quickly to accommodate the new extreme tempo values being presented, but the opposite range anchor does not recede, even though these extreme values are no longer being presented. This tendency to rapidly extend the range but only slowly retract the range has long been noted in the contextual judgment literature (Parducci, 1956). The lack of a significant interaction of context effects on tempo judgments with song familiarity suggests that these effects are independent of familiarity.

In Experiment 1, we demonstrated that the most pleasant tempo at which Beatles music is played changes with the manipulation of contextual tempos: The most pleasant tempo was faster in the fast context than in the slow context. This pattern of effects is consistent with previous research in other domains (Riskey et al., 1979; Wedell \& Pettibone, 1999; Wedell et al., 2005). Ideal point shifts in Experiment 1 appeared to exhibit some asymmetry. First, even in the between-subjects condition that was designed to maximize context effects, the ideal did not move below the original tempo value, whereas it moved quite strongly to a faster tempo value. Furthermore, carryover effects from the within-subjects design appeared asymmetric as well for pleasantness ratings. The most pleasant target tempo readily shifted to a faster tempo when the initial context was slow. However, the most pleasant target tempo did not shift to a significantly slower tempo when the initial context was fast. We investigated this asymmetric finding further in Experiment 2.

Somewhat surprisingly, familiarity was not a moderator of context effects for either tempo or pleasantness judgments. Previous research has shown fairly accurate remembered tempos for familiar songs (Levitin \& Cook, 1996) and an ability to discriminate the original tempo from a tempoaltered version that increases with familiarity with the song idiom (Honing \& Ladinig, 2009). These two findings provided a basis for the prediction that context effects might be reduced for participants who are more familiar with the song, since they would have a more stable representation in memory. This was not the case for either tempo or pleasantness judgments. Furthermore, the significant Familiarity $\times$ Target interaction on pleasantness judgments indicated that those more familiar with the song preferred a version that was faster than the original, whereas those less familiar with the song did not. Thus, there did not appear to be any special preference for the original for those more familiar with the song; indeed, the opposite was found.

The within-subjects manipulation of context also provided for a test of the judgment-mediated model of ideal point shifts. Because participants judged tempo and pleasantness in both fast and slow contexts, indices of contrast on tempo judgments and of most pleasant target tempo on pleasantness judgments could be calculated and compared. If contextual shifts in judged tempo cause corresponding changes in pleasantness as described in Eq. 2, then there should be a positive correlation between magnitude of contrast and magnitude of assimilation of ideals. However, this correlation was very small and not significant, consistent with prior research on judgments of schematic faces (Pettibone \& Wedell, 2007). Regression analyses support the conclusion that the lack of correlation was not due to differences in the slopes of judgment functions between and within participants.

One interpretation of the lack of correlation between these two effects is simply that the judgment-mediated model fails to account for shifts in ideals. Alternatively, it may be that the judgment-mediated model fails only to account for shifts in ideals when contexts change within subjects, possibly because of different factors affecting carryover for these two types of judgments. The fit of the judgmentmediated model to the data described in Table 3 supports this alternative interpretation. The judgment-mediated 
model predicted shifts in ideal points well for the first distribution judged, but not for the second distribution judged. The reduced correlations between individual and group ratings for the second distribution (shown in Fig. 5) suggests that participants may be somewhat idiosyncratic in whether they shift ideal points with the shift in context.

\section{Experiment 2}

In Experiment 2, a faster tempo Beatles song was used to investigate ideal point shifts in tempo preference. Paperback Writer $(P B W)$ was chosen because it has an original tempo speed of $156 \mathrm{bpm}$ (Leonard, 1994). We tested whether a faster song would produce more symmetrical ideal-point shifts. If participants do not shift toward slower than normal tempo values in the slow context using a faster tempo song, then it may be that musical stimuli produce inherently asymmetrical context effects. Such asymmetry may be the result of a generally positive correlation between tempo and liking for the song (LeBlanc et al., 1988, although see Geringer, 2010, for an exception). On the other hand, if participants shift equally toward slower and faster than toward original tempo values, we will conclude that musical stimuli can indeed produce symmetrical context effects for tempo. Another possibility is that the asymmetry might reverse, with participants more willing to shift to a slower tempo than a faster tempo for fast-paced music. This result would be consistent with Geringer, who found a preference for faster versions of slow paced songs and slower versions of fast paced songs. It would also be consistent with Moelants' (2002) assertion that there may be a preferred tempo centered near $120 \mathrm{bpm}$.

\section{Method}

The method of Experiment 2 was identical to that of Experiment 1 , except for the use of a different Beatles song. Instead of the relatively slow song $S P$ (95 bpm), a faster song $P B W$ (156 bpm) was used. A 13-s clip was taken from approximately 0:13 to 0:26 in the song and included the lyrics, "Dear sir or madam will you read my book? It took me years to write will you take a look? It's based on a novel by a man named Lear, and I need a job so I want to be a paperback writer." All other facets of the procedure and design were the same as in Experiment 1 except that a smaller number of participants, $N=$ 51 , was recruited.

\section{Results}

Between-subjects comparisons As in Experiment 1, participants were assigned to either the slow or fast tempo context for their first two sets of ratings so that comparisons between contexts were between subjects for these data. Figure 6 presents the mean ratings for tempo (panel A) and pleasantness (panel B), along with model fits. As in Experiment 1, large context effects were observed for both tempo and pleasantness ratings that are consistent with predictions. Moreover, the shifts in ideals shown in panel B of Fig. 6 appear to be more symmetric in nature, shifting below the original tempo value (0) as well as above it.

A 2 (context) $\times 5$ (target) repeated measures ANOVA was conducted on tempo ratings of the five target tempos. The significant main effect of context, $F(1,49)=88.4$, partial $\eta^{2}=$ $0.643, p<.001$, reflected the predicted contrast effect in which the same tempo was rated faster in the slow context than in the fast context. The only other significant effect was the main effect of target, $F(4,196)=72.2$, partial $\eta^{2}=0.596, p<.001$, which again reflected high sensitivity to tempo differences.

A 2 (context) $\times 5$ (target) repeated measures ANOVA was conducted on pleasantness ratings of the five target tempos. The significant main effect of context, $F(1,49)=10.4$, partial $\eta^{2}=0.175, p<.001$, reflected higher pleasantness rating for the target tempos in the slow context. The significant main effect of target, $F(4,196)=6.2$, partial $\eta^{2}=0.112, p<.001$, reflected the predicted curvilinear relation between pleasantness and tempo for the target values. The significant Context $\times$ Target interaction, $F(4,196)=15.4$, partial $\eta^{2}=0.239, p<$ .001 , reflected the predicted crossover interaction in which the peak of the pleasantness rating function is shifted to a faster target value in the fast context than in the slow context. As predicted, the linear component of the interaction was significant, $F(1,49)=24.5$, partial $\eta^{2}=0.333, p<.001$. To assess ideal point shifts more directly, most pleasant target tempos were computed for each participant as in Experiment 1. A $t$ test on this measure was significant, $t(49)=3.48, p<.01$, with the most pleasant target tempo higher in the faster context $(M=6.39)$ than in the slower context $(M=-0.36)$. As demonstrated in panel B of Fig. 6, the target tempo of $-6.0 \%$ is clearly rated most pleasant in the slow context, indicating a shift toward a slower tempo.

As in Experiment 1, familiarity varied widely across participants $(M=3.56, S D=2.87)$; however, the modal familiarity rating was 1.0 , and the median familiarity was 2.0. Because of the highly skewed nature of familiarity ratings, we used this variable in the ANOVAs described previously in two ways. In one set of analyses we included it as a continuous variable, and in the other, we included it as a dichotomous variable based on a median split. In none of these analyses was there a main effect of or interaction with the familiarity variable. Hence, once again there is no evidence of familiarity moderating contextual effects on tempo or pleasantness judgments.

Within-subjects comparisons Figure 7 presents the data for the within-subjects comparisons of context effects, along 
Fig. 6 Mean ratings of tempo (a) and pleasantness (b) for target tempos (circles) and context tempos (squares) in slow- and fast-tempo contexts in Experiment 2 using the Beatles song Paperback Writer. Results demonstrate contrast effects on tempo ratings and assimilation of ideals for pleasantness ratings
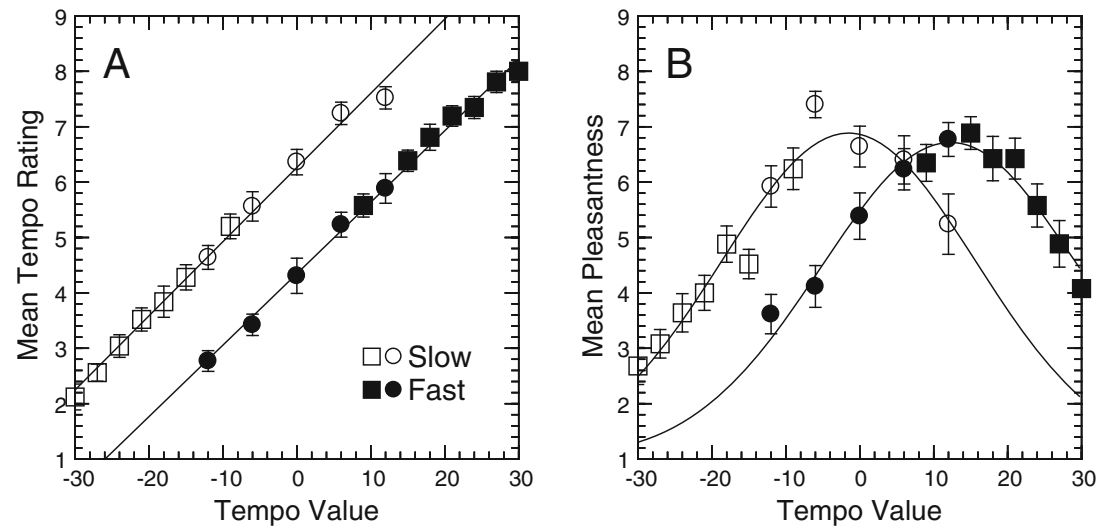

with the model fits (fit parameters are shown in Table 2). Strong contrast effects on tempo ratings were found for these within-subjects comparisons (left panels), but again there were clear carryover effects. Unlike in Experiment 1, the pleasantness ratings appear to show a clear difference in the peaks of the functions for both order conditions.

Parallel analyses were conducted on tempo ratings across the two blocks so that context was a within-subjects factor. A 2 (order of context) $\times 2$ (context) $\times 5$ (target) repeated measures ANOVA conducted on tempo ratings of the target tempos revealed a large effect of context, $F(1,49)=45.7$, partial $\eta^{2}=0.483, p<.001$, reflecting a strong contrast effect on tempo ratings when context was manipulated within subjects. Once again the within-subjects contrast was quite large (a mean difference of 0.93), although it was only about half the size of the between-subjects contrast (a mean difference of 1.94), reflecting carryover effects of context from the first block. Further evidence of carryover was found in the significant main effect of order of context, $F(1,49)=45.5$, partial $\eta^{2}=0.481, p<.001$, in which target ratings were higher when the slow context was presented first. Other than a significant main effect of target, $F$ $(4,196)=127.0$, partial $\eta^{2}=0.722, p<.001$, no other effects were significant.

A parallel $2 \times 2 \times 5$ repeated measures ANOVA conducted on pleasantness ratings of the target tempos revealed
Fig. 7 Mean ratings of tempo (left panels) and pleasantness (right panels) for target tempos (circles) and context tempos (squares) in slow- and fasttempo contexts in Experiment 2 as a function of context order. Ratings in each panel are from the same participants reflecting within-subjects context effects. Range-frequency fits are shown for tempo ratings and Gaussian ideal point fits are shown for pleasantness ratings
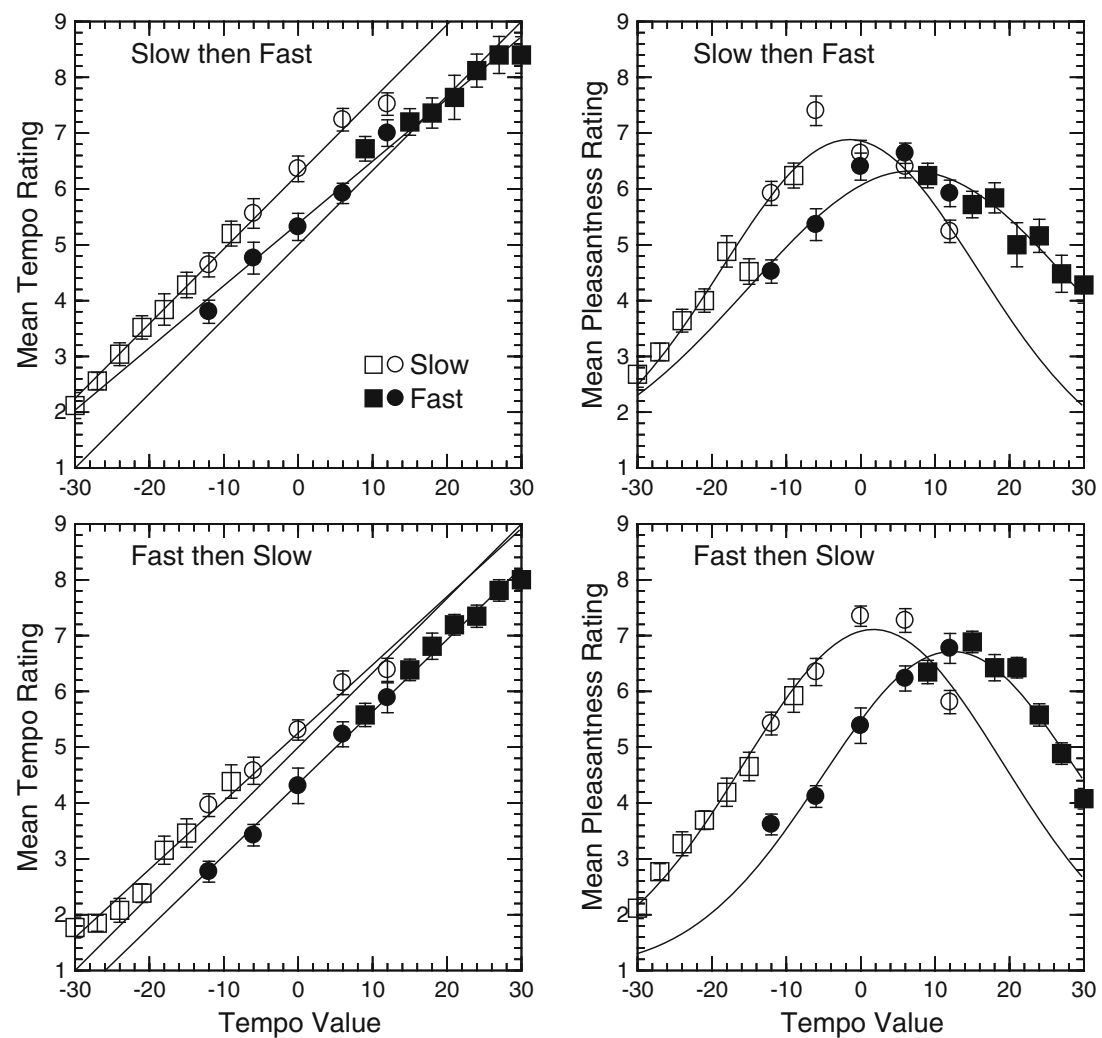
a large Context $\times$ Target interaction, $F(4,196)=13.2$, partial $\eta^{2}=0.212, p<.001$, reflecting the crossover interaction predicted by a shift in ideals toward contextual values as described in a significant linear component, $F(1,49)=15.0$, partial $\eta^{2}=0.234, p<.001$. The lack of a significant main effect of order of context is consistent with the more symmetric shifts of ideals found in Experiment 2. Transfer effects from the first context are reflected in the significant Order $\times$ Target interaction, $F(4,196)=4.8$, partial $\eta^{2}=0.089$, $p<.001$. Additional significant effects included a main effect of context, $F(1,49)=16.6$, partial $\eta^{2}=0.253, p<.001$, reflecting higher overall pleasantness ratings in the slow context and a main effect of target, $F(4,196)=16.9$, partial $\eta^{2}=$ $0.407, p<.001$, reflecting the predicted single peaked shape of the pleasantness rating function as described by the quadratic trend, $F(1,49)=41.0$, partial $\eta^{2}=0.456, p<.001$. No other effects were statistically significant.

To illustrate significant preference reversals for these withinsubjects data, we conducted comparisons of pleasantness ratings for $-6 \%$ and $12 \%$ targets in each context. For the slow context, participants rated $-6 \%$ significantly more pleasant than $12 \%, t(50)=2.99, p<.01\left(M_{\text {Slow }}=6.86\right.$ and $\left.M_{\text {Fast }}=5.53\right)$. However, these same participants rated $-6 \%$ significantly less pleasant than $12 \%$ in the fast context, $t(50)=-4.51, p<.001$ $\left(M_{\text {Slow }}=4.73\right.$ and $\left.M_{\text {Fast }}=6.35\right)$.

Model fits The model fitting procedures described in Experiment 1 were used again to augment the analyses for Experiment 2. Table 2 presents the range-frequency parameters fitted to the tempo judgment data, with the corresponding model fits shown in the left panels of Figs. 6 and 7. The model provided a good fit to the empirical values $\left(R^{2}=.992\right.$ for slow to fast, and $R^{2}=.993$ for fast to slow). Once again, the model parameters indicate that the nature of the carryover effects on tempo judgments appears to be due to the tendency to hold onto an extreme range anchor after the context has changed. Although this tendency held true in both conditions, it was somewhat reduced for the fast to slow task order.

The ideal-point model of Eq. 4 was once again fit to the data averaged across participants within conditions. The model fit the data fairly well $\left(R^{2}=.940\right.$ for slow to fast order and $R^{2}=.977$ for fast to slow order). As shown in Table 2, both order conditions produced fairly substantial differences in ideal points for slow and fast contexts. Unlike Experiment 1, in which this difference was substantially smaller in the fast to slow context, the reverse was true, with the differences in inferred ideal points somewhat smaller in the slow to fast context.

Table 3 also shows the same type of analysis conducted in Experiment 1 to determine how well the judgmentmediated model predicted ideal point differences. Once again, predictions were accurate for the first context encountered, but predictions were less accurate for the second context encountered.

Individual differences analyses As in Experiment 1, the significant within-subjects effects of context made it possible to test the hypothesis that differences in judged tempo mediate the effects of differences in the most pleasant target tempo. Measures of contrast differences and most pleasant target tempo differences were calculated in the same way as in Experiment 1. Consistent with Experiment 1 results and inconsistent with the hypothesis of judgment-mediated effects on pleasantness, the correlation between these two effects was close to zero and not significant, $r=.08$. This correlational analysis was followed up by regression analyses (as described in Experiment 1), which included measures of each participant's average slope across the two contexts and the difference in slopes across the two contexts based on the target tempo ratings. In none of these regression analyses was the model $R^{2}$ or the change in $R^{2}$ with additional terms significant. Thus, the lack of correlation between these two measures was not moderated by individual slope differences or averages.

\section{Discussion}

Like in Experiment 1, in Experiment 2 we demonstrated strong and significant context effects on tempo and pleasantness ratings consistent with predictions generated from Eqs. 1 and 4. As in Experiment 1, participants' song familiarity did not moderate these context effects, although there was a tendency for participants to be less familiar with the Beatles song used in this experiment. Furthermore, although strong within-subjects context effects were found for both tempo and pleasantness ratings, these contextual shifts were uncorrelated, indicative of the independence of these two contextual processes. The analysis shown in Table 3 indicates that like Experiment 1, the judgment-mediated model predictions do well for the initial context but not for the context encountered second.

In addition to replicating Experiment 1's results with another song, a major focus of Experiment 2 was to determine whether the asymmetry of ideal-point shifts found in Experiment 1 would be reduced when a song with a faster original tempo was used. There is abundant evidence of a general preference for faster tempo songs across a wide range of ages (LeBlanc et al., 1988). Thus, when manipulating tempos of a slow song, it may be relatively easy to shift pleasantness toward a faster version, but more difficult to shift pleasantness toward a slower version. In Experiment 2, we used a much faster tempo song ( $P B W$ at $156 \mathrm{bpm})$ than in Experiment 1 ( $S P$ at $95 \mathrm{bpm})$. With a faster original tempo, shifting tempo pleasantness toward slower versions 
may be easier. Consistent with this prediction, the peak of the pleasantness function shifted to a $6 \%$ slower tempo in the slow context, as shown in panel B of Fig. 6. Therefore, we do not believe that ideal-point shifts for tempos must be inherently asymmetric, although they may tend to be so for slower songs. Because we found no strong evidence for the reversal of the asymmetry, our results do not support a hypothesis based on the idea that shifts are easier when made toward a preferred tempo of $120 \mathrm{bpm}$ (Moelants, 2002).

\section{Experiment 3}

In Experiments 1 and 2, we demonstrated strong effects of manipulating a song's tempo on evaluations of tempo and pleasantness for different versions of the same song. These effects occurred regardless of familiarity with the songs. Although in Experiments 1 and 2 we demonstrated robust effects of contextual manipulations of a given song's tempo, they do not address the issue of whether these context effects will occur when manipulation of contextual tempos occurs for a song that is different from the target song being judged. For example, one might ask whether judgments of $S P$ would be affected by listening to speeded up or slowed down versions of $P B W$. Such effects would depend critically on what information is recruited into the relevant context for evaluations.

It has long been known that context effects generally do not occur when contextual stimuli are categorically different from target stimuli. For example, Brown (1953) found that evaluations of lifted weights were unaffected by having participants lift other objects not associated with the experimental stimuli (such as a tray). A number of researchers have demonstrated diminished effects of context as a function of the perceived similarity of contextual and target stimuli, as determined by categorical classification, relevance to the task, or magnitude differences (Sarris, 1976; Zellner et al., 2003). For example, Parducci, Knoble, and Thomas (1976) found that context effects on judgments of square sizes were largely independent of the manipulation of circle sizes and vice versa, implying that squares recruited other square sizes for contextual comparison and circles recruited other circle sizes for contextual comparisons. However, a key issue that arises from this perspective concerns the conditions under which judges perceive target and contextual stimuli to be from different categories. For example, is the relevant category for determining contextual recruitment in the present case simply any song, a Beatles song, a Beatles song with a similar tempo, or a Beatles song with a similar tempo and similar characteristics? This issue is not easily resolved a priori. For example, Rota and Zellner (2007) demonstrated that although novice participants showed contrast effects across different flower types, those with expertise in flowers placed the target and contextual stimuli (irises and orchids) into different categories and thereby showed no significant contrast.

Although determining the characteristics of a song that result in generalizing the effects of context is an important issue in its own right, it may also be useful theoretically in distinguishing the processes underlying the two types of context effects we have observed. In Experiment 3, we tested whether the contexts recruited for tempo judgments and pleasantness judgments differ, a result that would support separate mechanisms for these two types of context effects. On the basis of previous research, one might argue that the relevant context for judging the tempo of a song consists of other songs of similar tempo one has recently heard. On the other hand, if judgments of pleasantness are based on a comparison to a prototypical value for a given song, then one might expect a song-specific effect in which only contextual manipulations of the same song will produce shifts in ideals. In Experiment 3, we tested how context effects transfer to other songs by manipulating contextual tempos for one Beatles song and evaluating the effect of this manipulation on that same song along with three other Beatles songs.

Figure 8 presents a flow diagram of Experiment 3. Participants were instructed that they would be judging musical clips from four Beatles songs, $P B W$, Something (SOM), She Loves You (SLU), and The Long and Winding Road (LWR). They were then presented with the unaltered versions of each to be sure that everyone had an idea of what these sounded like regardless of prior familiarity. They then proceeded to 40 tempo judgment trials, with every other trial being sampled from the contextual set or the target set. The contextual set was manipulated between subjects and consisted of fast or slow versions of just one song (either $P B W$ or $S O M)$. The target set was manipulated within subjects and consisted of the five target clips $(-12 \%,-6 \%, 0,6 \%$ and $12 \%$ ) of each of the four songs ( $P B W, S O M, S L U, L W R)$. It should be noted that $S L U$ was selected to have a bpm that was very similar to the contextual song $P B W$ (a fast tempo song), and that $L W R$ was selected to have a bpm that was very similar to the contextual song SOM (a slow tempo song). After making the 40 tempo judgments, participants proceeded to 40 pleasantness judgments, with the contextual and target clips once again randomly sampled on every other trial. After making these judgments, participants listened to six pairings of the original clips and judged the similarity of these songs in order to validate the similarity manipulation. In summary, Experiment 3 was designed to manipulate the contextual tempos for one song and to examine the impact of this manipulation on tempo and pleasantness judgments of target tempos for (a) that same song, 
Fig. 8 Flow diagram describing procedural details of Experiment 3

\section{Experiment 3}

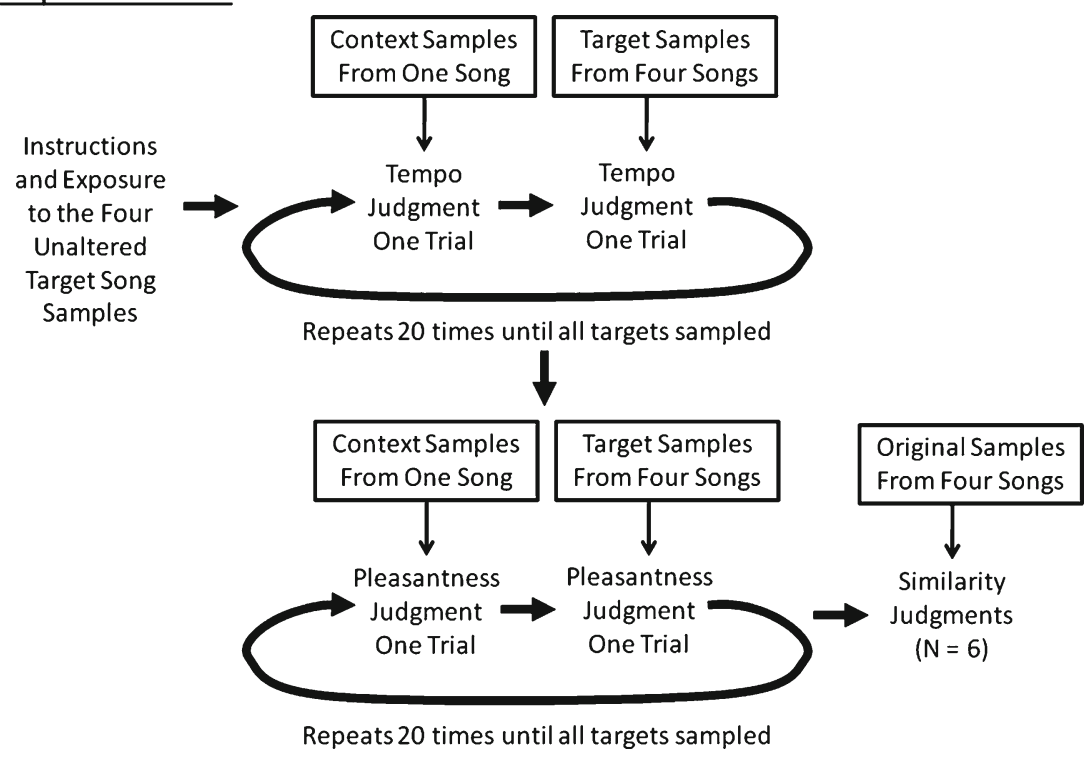

(b) a similar tempo song, and (c) two dissimilar tempo songs.

On the basis of this design, we generated a number of predictions. First, following the literature on contextual contrast, we predicted that contrast effects on tempo ratings would occur when the context consisted of the same song or a song with a similar original tempo. Thus, we expected contrast effects for both $P B W$ and $S L U$ targets when $P B W$ was the context song but not when $S O M$ was the context song. Conversely, we expected contrast effects for $S O M$ and $L W R$ targets when SOM was the context song but not when $P B W$ was the context song. Predictions for pleasantness ratings depended on which mechanism was assumed to drive these effects. If ideal point shifts are mediated by tempo judgments, then one would expect the same pattern of effects to occur for pleasantness ratings. On the other hand, if pleasantness ratings follow from a prototype model of memory traces of the specific song, then we should find only song-specific pleasantness effects in which $P B W$ targets show an ideal point shift when $P B W$ is the context song and, similarly, in which $S O M$ targets show an ideal point shift when $S O M$ is the context song. Finally, if pleasantness shifts are related to more general shifts in levels of arousal induced by fast or slow context tempos, then effects might be expected to generalize to all targets. It should be noted that Holbrook and Anand (1990) proposed this type of generalized arousal mechanism and found some support for it in that shifts in preferred tempo reflected changes in arousal state induced by performing different cognitive tasks prior to listening. Because researchers have demonstrated that listening to fast-paced music leads to increases in arousal indicators such as heart rate and performance in stationary cycling and running (Edworthy \& Waring, 2006; Waterhouse, Hudson, \& Edwards, 2010), the idea of a generalized arousal effect driving ideal point shifts seems plausible.

\section{Method}

Participants and design Participants were 130 undergraduates at the University of South Carolina who received course credits for volunteering to participate. They were assigned to one of four conditions generated by the $2 \times 2$ factorial combination of two between-subjects variables, contextual song ( $P B W$ or $S O M$ ), and contextual tempo (slow or fast). The experimental design also included two factorally combined within-subjects variables, target song ( $P B W, S L U$, $S O M, L W R)$ and target tempo level $(-12 \%,-6 \%, 0 \%, 6 \%$, and $12 \%$ of the original). Across the 80 trials, a contextual song was always presented and judged prior to the presentation and judgment of a target song, as depicted in Fig. 8. Contextual and target clips were randomly sampled without replacement for each participant. Thus, on odd trials the participant would always hear a version of the context song (sampled from the contextual range), and on even trials the participant would always hear a version of one of the four target songs (sampled from the target range). On the first 40 trials, participants rated the tempo of each song, and on the next 40 trials, they rated the pleasantness of the tempo of each song. The dependent variables were tempo and pleasantness ratings recorded on a 9-point scale.

Materials and apparatus All experimental materials were presented on desktop computers with 17-in. monitors and headphones. The same 13-s clip from $P B W$ was used again. 
Additionally, clips of approximately 13 or $14 \mathrm{~s}$ were taken from the songs $S L U, L W R$, and $S O M$. The clip from $S L U$ was taken from approximately $0: 12$ to $0: 25$ and included the following lyrics, "You think you've lost your love, well I saw her yesterday. It's you she's thinking of, and she told me what to say." The clip from SOM was taken from approximately 0:05 to 0:19 in the song and included the following lyrics, "Something in the way she moves, attracts me like no other lover." The clip from $L W R$ was taken from approximately 0:50 to 1:04 and included the following lyrics, "That the rain washed away, has left a pool of tears."

Context song $P B W$ was matched closely in tempo with target song $S L U$ (156 and $158 \mathrm{bpm}$ ), whereas context song $S O M$ was matched closely in tempo with target song $L W R$ (66 and $66 \mathrm{bpm}$ ). Thus, each context song had one target song that was a direct match ( $P B W$ with $P B W$ and $S O M$ with $S O M)$, one target song that was a close match in tempo ( $P B W$ with $S L U$ and $S O M$ with $L W R$ ), and two target songs that were a mismatch in tempo ( $P B W$ with $S O M$ and $L W R$, and $S O M$ with $P B W$ and $S L U$ ). Table 1 presents the bpm for the different contextual and target songs. In the slow context, the contextual clips $-30 \%$ and $-27 \%$ were presented eight times each, and clips $-24 \%,-21 \%,-18 \%$, and $-15 \%$ were presented six times each. In the fast context, the contextual clips $30 \%$ and $27 \%$ were presented eight times each, and clips $24 \%, 21 \%, 18 \%$, and $15 \%$ were presented six times each. Note that because fast and slow contexts were determined by the percent slowing or speeding up of the original tempo, the actual tempos in bpm differ across contextual and target songs.

Procedure Participants were tested in groups of up to four in a large laboratory room with computers spaced about $2 \mathrm{~m}$ apart. Instructions informed them that they were going to listen to several clips from Beatles songs that would be played at different tempos. They were instructed that after each short clip was played, they would be asked to rate it either in terms of how fast or slow it sounded or in terms of how pleasant the tempo for that song sounded to them. They then listened to the original tempo versions of the four song samples in order to familiarize them with the songs being presented. They were then instructed that in the first phase they would be judging tempo using a 9-point scale. After 40 judgment trials in the first phase, they were instructed that they should now judge how pleasant the song's tempo sounded to them using a 9-point scale. After 40 judgment trials in the second phase, they were asked to rate how similar the pairs of songs sounded to them using the scale $1=$ not at all similar to $9=$ very very similar. They then were presented the six pairs of original song samples in random order. Finally they rated how familiar they were with the music clips they had just listened to on a 9-point scale.
Results

Mean similarity ratings were compared to validate the manipulation of similarity of target and contextual songs. As expected, the rated similarity was high for $P B W$ to $S L U(M=6.75)$ and for $S O M$ to $L W R(M=$ 6.76), and these did not differ significantly from one another, $t(129)=-0.07$. All other similarity comparisons were relatively low: $M=2.91$ for $P B W$ to $L W R, M=$ 2.85 for $P B W$ to $S O M, M=2.78$ for $S O M$ to $S L U$, and $M=2.78$ for $L W R$ to $S L U$. These means were submitted to a one-way repeated measures ANOVA and were found not to differ significantly from one another, $F(3,387)=0.24$. Thus, contextual songs were each identical to one of the target songs, of high similarity to another, and of low similarity to the other two target songs in terms of tempo.

Evaluation of contextual songs The experimental design allowed us to conceptually replicate Experiments 1 and 2 by examining how ratings for a song are affected by contextual manipulation of that same song. Figure 9 presents the rating functions for $P B W$ and $S O M$, with tempo ratings shown on the left and pleasantness ratings shown on the right, along with model fits of Eqs. 1 and 4. Replicating previous findings, the tempo ratings show clear contextual contrast, with rated tempo higher in the slow than in the fast context. This effect was supported by $t$ tests comparing the mean rating of target songs for fast and slow contexts. For $P B W$, tempo ratings were significantly higher in the slow context $(M=7.10)$ than in the fast context $(M=4.64), t(63)=9.59, p<.001$. For $S O M$, tempo ratings were also significantly higher in the slow context $(M=4.19)$ than in the fast context $(M=2.38), t(63)=$ $7.57, p<.001$.

Table 2 presents the inferred values of stimuli anchoring the range inferred from the fit of Eq. 1 to the data. Model fits were again quite good $\left(R^{2}=.976\right.$ for $P B W$, and $R^{2}=.987$ for $S O M$ ). Thus, for each song, the shift in tempo ratings was well described by a shift in the subjective range. One difference from previous fits to data of Experiments 1 and 2 was that more extreme values were used to define the range. For the faster tempo $P B W$, the value of the subjective minimum went as low as -52.56 , much lower than in Experiment 2. For the slower tempo SOM, the value of the subjective maximum went as high as 88.96 . These extreme range values are likely due to participants' using the full set of target songs as part of their context.

Also consistent with Experiments 1 and 2, the ideal pleasantness value shifted with context, reflecting an assimilation of the ideal point toward contextual values. To test for these differences, the most pleasant target tempo was computed as in Experiments 1 and 2. The shift in ideals was supported by $t$ tests comparing the most pleasant target 
Fig. 9 Mean ratings of tempo (left panels) and pleasantness (right panels) for target tempos (circles) and context tempos (squares) in slow- and fasttempo contexts in Experiment 3 using the Beatles song Paperback Writer (top panels) or Something (bottom panels). Results demonstrate contrast effects on tempo ratings and assimilation of ideals for pleasantness ratings. Rangefrequency fits shown for tempo ratings and Gaussian ideal point fits shown for pleasantness ratings
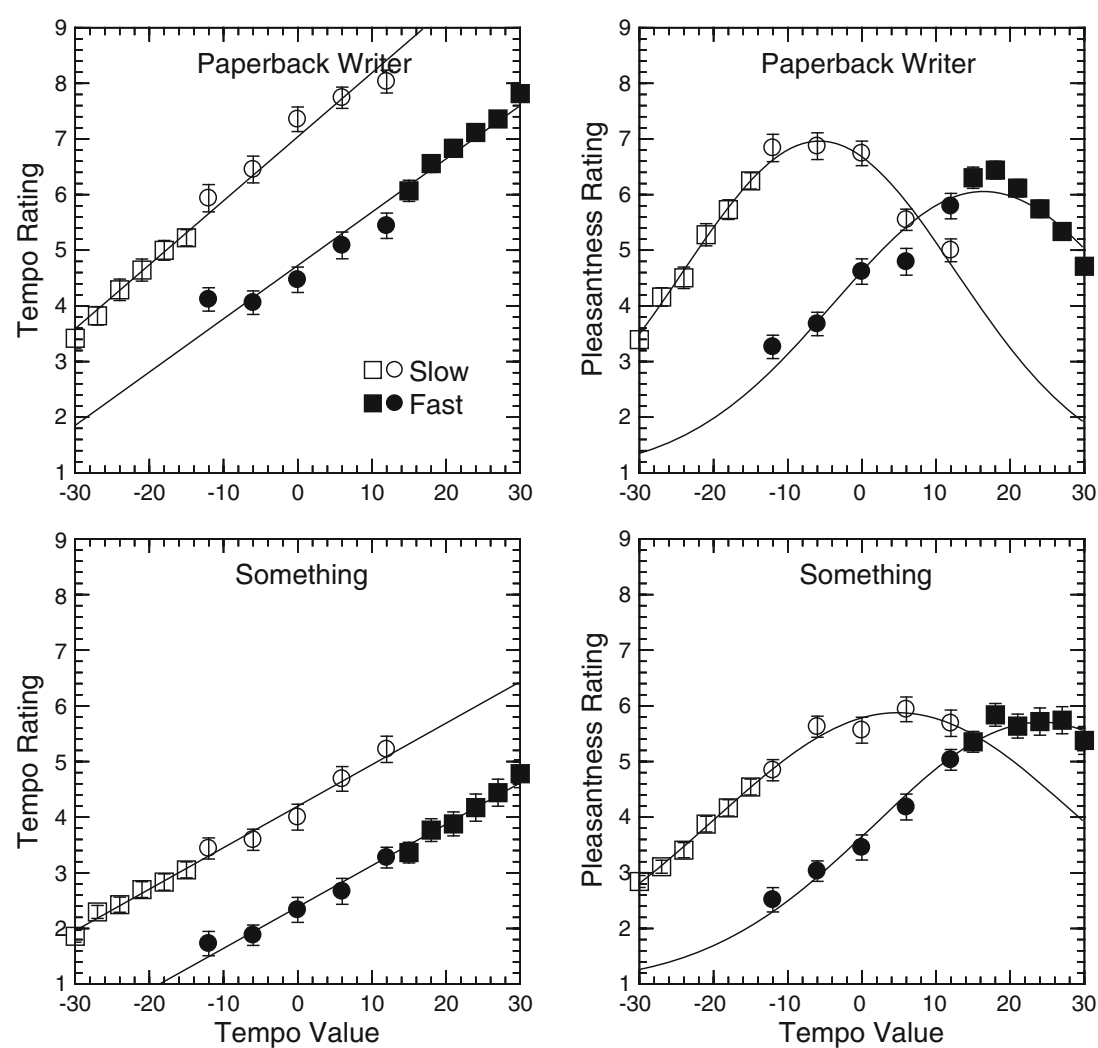

tempo for fast and slow contexts. For $P B W$, the most pleasant target tempo was significantly lower in the slow context $(M=-2.03)$ than in the fast context $(M=7.13), t(63)=-5.99$, $p<.001$. For $S O M$, the preferred target value was also significantly lower in the slow context $(M=2.56)$ than in the fast context $(M=7.00), t(63)=-2.74, p<.01$. The inferred ideals from fitting the ideal-point model of Eq. 4 were generally more extreme than the most pleasant target tempos, as shown in Table 2 . The ideal-point model fit reasonably well $\left(R^{2}=.976\right.$ for $P B W$, and $R^{2}=.987$ for $S O M$ ). It should be noted that the contextual shifts in ideal points were larger than those found in Experiments 1 and 2. This is not surprising considering that, using a song-specific context, there were 20 contextual presentations for every five target presentations in Experiment 3, as compared with seven contextual presentations for every five target presentations in Experiments 1 and 2.

Evaluation of target songs Using the key comparisons in Experiment 3, we examined how context effects depend on the match of the target to the context. Song-specific effects would occur if the contextual effect occurred only when the context and target were from the same song. Similarity transfer would occur if the context effects from a song transferred to a similar song but not to dissimilar songs. Generalized context effects would occur if all target songs showed similar contextual effects. We will first examine these results for tempo judgments and then for pleasantness judgments.

Figure 10 shows the tempo ratings of target songs when either $P B W$ was presented at fast or slow tempos (top row) or when $S O M$ was presented at fast or slow tempos (bottom row). The pattern shown in Fig. 10 is quite clear: Tempo context applies to the same song target, transfers to the target song of similar base tempo, but does not transfer to target songs of dissimilar base tempos. Thus, in the top row, $P B W$ and $S L U$ targets show strong contrast effects but $L W R$ and $S O M$ targets do not when $P B W$ is the context song. Conversely, in the bottom row, SOM and $L W R$ targets show strong contrast effects, but $P B W$ and $S L U$ targets do not when $S O M$ is the context song. This result was statistically confirmed by the significant three-way interaction when the mean rating of each set of targets was analyzed in a 2 $($ context song) $\times 2$ (context tempo) $\times 4$ (target song) ANOVA, $F(3,378)=38.31$, partial $\eta^{2}=0.233, p<.001$. A simple effects analysis clarifies how the Context Tempo $\times$ Target Song interaction differs for the two context songs. When $P B W$ was the context song, the effect of context tempo was significant for $P B W$ and $S L U$ targets $(p<.001)$, but was not significant for either $L W R$ or SOM targets ( $p>$ $.05)$. The opposite was true when $S O M$ was the context song: The effect of context tempo was not significant for $P B W$ and $S L U$ targets $(p>.05)$, but it was significant for 
Fig. 10 Mean tempo ratings across five different target tempos for four different target songs as a function of context (fast or slow) and context song (Paperback Writer $[P B W]$ or Something $[S O M])$. The other target songs were She Loves You $(S L U)$ and The Long and Winding Road (LWR). Contrast effects were found when the target song was the same as or similar to the context song
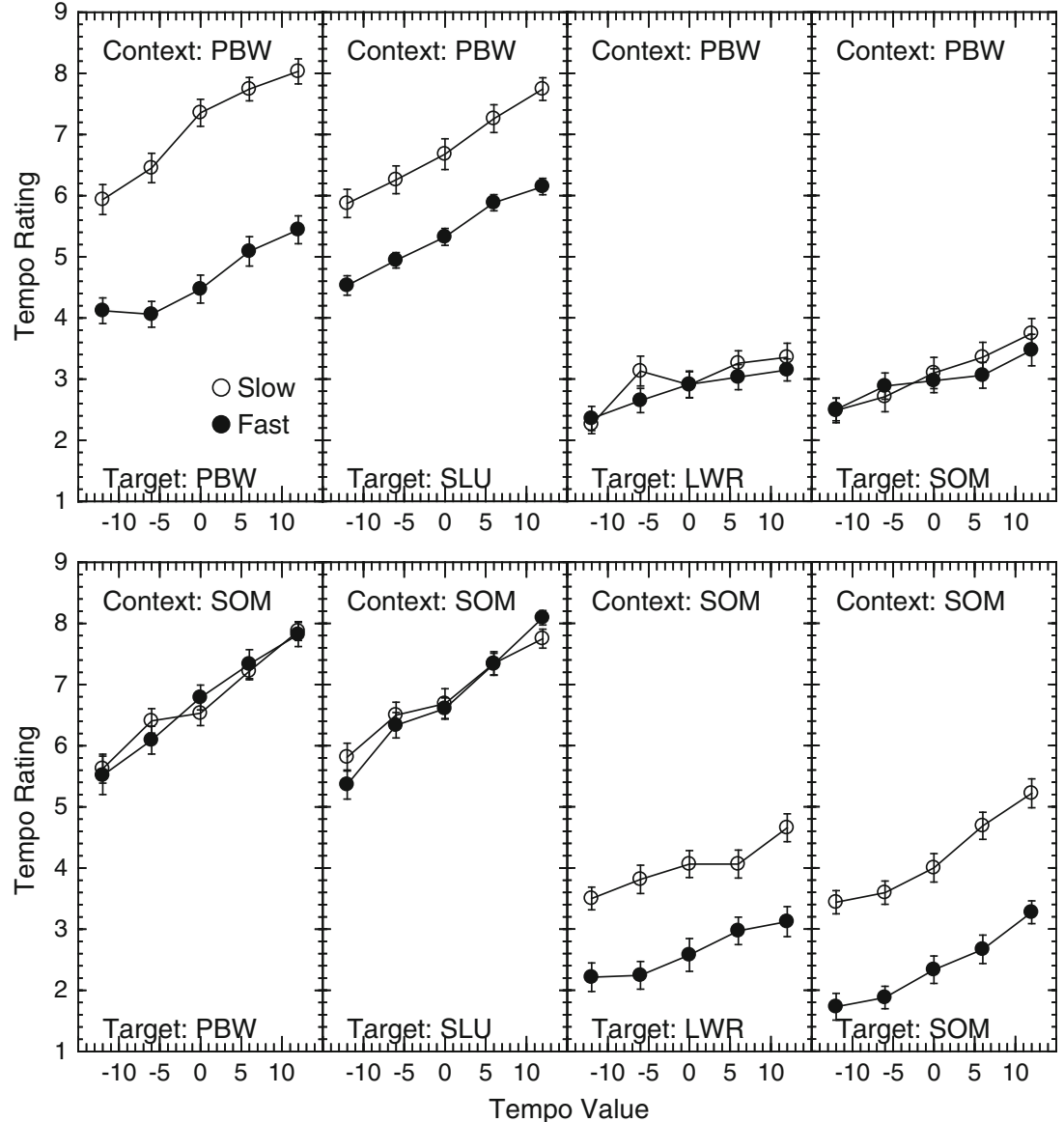

$L W R$ and SOM targets $(p<.001)$. This pattern is clearly in line with similarity-based transfer effects and not songspecific or song-general effects.

Figure 11 shows the pleasantness ratings of target songs when either $P B W$ was presented at fast or slow tempos (top row) or when $S O M$ was presented at fast or slow tempos (bottom row). The predicted shift in ideal point should be reflected in a crossover interaction, with the rating function across targets sloping down in the slow context and sloping up in the fast context. The pattern of context effects on ideals shown in Fig. 11 is best described as song specific, with contextual shifts in ideals occurring for the same song but not for similar or dissimilar songs. This pattern is clearest in the top row of Fig. 11, since only the $P B W$ targets showed any contextual shift in pleasantness ratings with manipulation of the tempo of $P B W$ context. The bottom panel appears a bit more ambiguous, with the effects of $S O M$ context on $L W R$ targets appearing similar in form to the effects on the SOM targets, though much smaller. In our statistical analysis, the most pleasant target tempo was submitted to a 2 (context song) $\times 2$ (context tempo) $\times 4$ (target song) ANOVA to test for shifts in ideal points with context. The three-way interaction was significant, $F(3,378)=7.98$, partial $\eta^{2}=0.059, p<.001$. Simple effects analyses demonstrated that the only significant shift in most pleasant target tempos occurred when target and context songs matched. Thus, most pleasant target tempo significantly shifted for $P B W$ targets when $P B W$ was the context song and similarly shifted for $S O M$ targets when $S O M$ was the context song. Note that overall ratings of pleasantness shifted for the LWR target song under the SOM context, but the most pleasant target tempo value did not significantly shift. Thus, the results for both contexts are consistent with the song specific hypothesis.

We explored the plausibility of the judgment-mediated model explanation of these data further by determining quantitative predictions like those described in Table 3. The results of this analysis are shown in Table 4. This analysis may be viewed with some caution, since the model fits used a data range restricted to the target values. First, we fit Eq. 4 to the data of Fig. 11 in order to infer ideal points in each condition. Two conditions proved problematic in that the function in the target range was fairly linear and so ideals were difficult to estimate ( $P B W$ targets for the fast $P B W$ context and SOM targets for the fast $S O M$ context). In both of these conditions, we had more stable estimates available based on the full range of data (see Fig. 9); however, we used the estimates from the restricted data so that 
Fig. 11 Mean pleasantness ratings across five different target tempos for four different target songs as a function of context (fast or slow) and context song (Paperback Writer $[P B W]$ or Something $[S O M])$. The other target songs were She Loves You (SLU) and The Long and Winding Road ( $L W R$ ). Assimilation of ideals occurred only when the target song was the same the context song
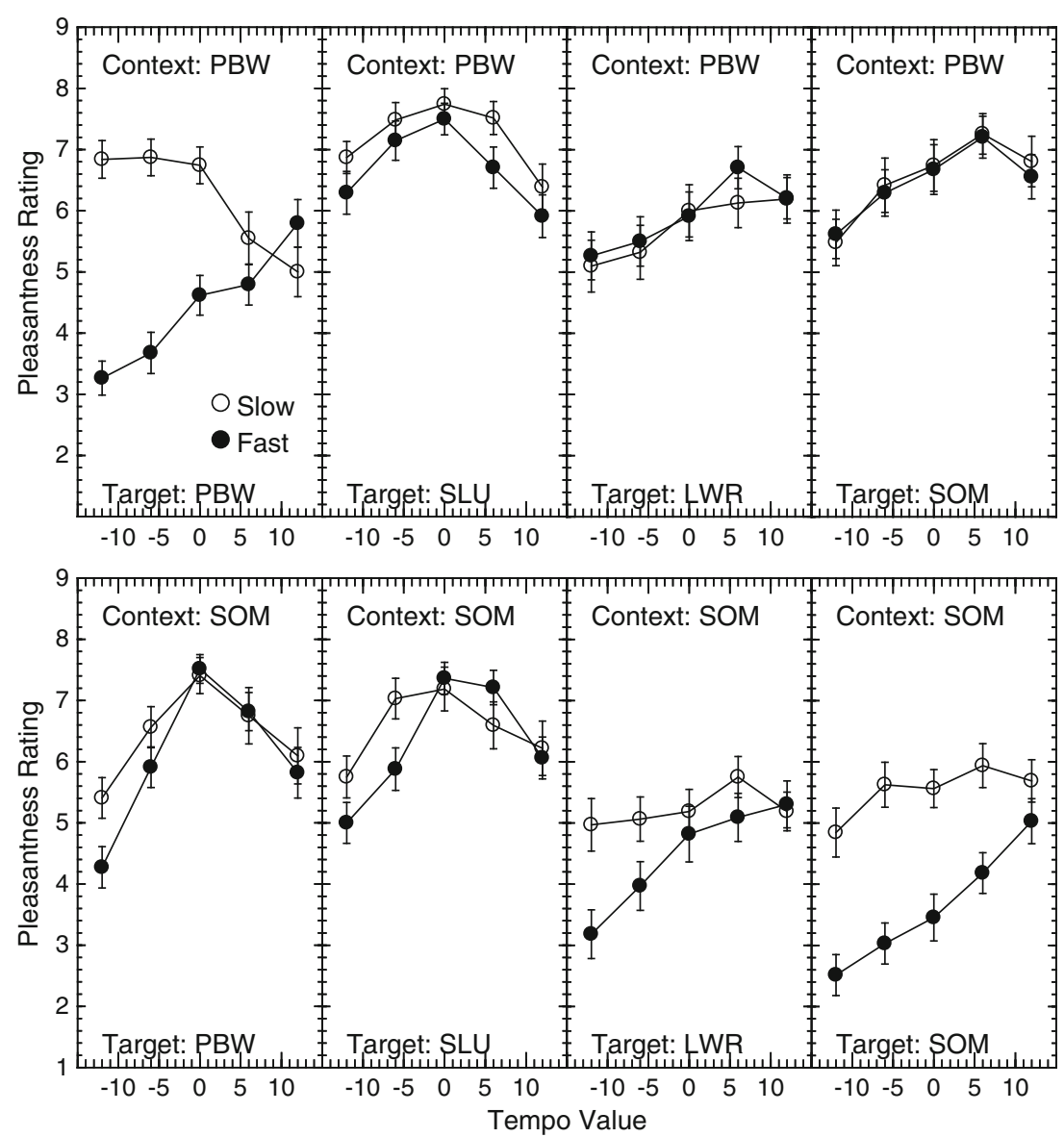

all comparisons were based on the same method. We then fit the range-frequency Eq. 1 to the data of Fig. 10, fitting $S_{M I N}$ and $\mathrm{S}_{\mathrm{MAX}}$ for each condition. We then generated predicted ideal points using a value of $\mathrm{J}_{\text {Ideal }}$ that averaged estimates of $\mathrm{J}_{\text {Ideal }}$ from fits to the inferred ideals.

As shown in Table 4, and consistent with results of Table 3, the judgment-mediated model did a reasonable job predicting the between-subjects effects of context when target song matched context song. In these cases, strong contrast effects on tempo judgments accompanied strong contextual shifts of ideal points for the pleasantness judgments. However, as expected, the judgment-mediated model incorrectly predicted large shifts in ideals when the target song was similar to the context song ( $S L U$ paired with $P B W$ and $L W R$ paired with $S O M$ ). The analysis shown in Table 3 reinforces the conclusions that the judgment-mediated model cannot adequately account for the pattern of data observed in Experiment 3 .

\section{Discussion}

In Experiment 3, we explored the degree to which the context effects on judgments of tempo and pleasantness demonstrated in Experiments 1 and 2 occurred when the target song differed from the context song. We considered three possibilities. The first, song-specific contextual processing, implies that context song tempos are recruited only when they reflect a variation of the song being judged, and thus context effects should occur only for variations of the same song. The second, similarity-driven contextual processing, implies that context effects generalize to other similar songs but not to dissimilar songs. The third, generalized contextual processing, implies that context effects occur across a wide range of songs, regardless of similarity. A generalized effect for tempo-related ratings might derive from physiological changes in arousal level linked to tempo (Edworthy \& Waring, 2006; Waterhouse et al., 2010).

We expected that context effects for tempo ratings would be similarity driven so that the manipulation of the tempo of a song would affect tempo ratings of target samples from that song along with target samples from other similar songs but not target samples from dissimilar songs. This prediction was based on the demonstration of similarity based contextual recruitment for contrast effects in the literature (Parducci et al., 1976; Sarris, 1976; Zellner et al., 2003). This prediction was largely borne out by the results of Experiment 3. Manipulation of the $P B W$ context affected tempo ratings of $P B W$ 
Table 4 Comparison of judgment-mediated model-predicted ideals with inferred ideals (Experiment 3)

\begin{tabular}{|c|c|c|c|c|c|c|c|}
\hline Context Song & Context Set & Target Song & $\mathrm{S}_{\mathrm{MIN}}$ & $\mathrm{S}_{\mathrm{MAX}}$ & $\mathrm{J}_{\text {Ideal }}$ & Predicted Ideal & Inferred Ideal \\
\hline$P B W$ & slow & $P B W$ & -66.78 & 20.75 & 6.20 & -9.61 & -8.21 \\
\hline$P B W$ & fast & $P B W$ & -59.33 & 71.23 & 6.20 & 25.95 & 23.86 \\
\hline$P B W$ & slow & $S L U$ & -72.9 & 28.32 & 5.96 & -9.89 & -1.01 \\
\hline$P B W$ & fast & $S L U$ & -62.7 & 52.23 & 5.96 & 8.85 & -1.23 \\
\hline$P B W$ & slow & LWR & -51.17 & 155.5 & 3.32 & 8.3 & 12.25 \\
\hline$P B W$ & fast & LWR & -55.34 & 188.24 & 3.32 & 14.75 & 10.09 \\
\hline$P B W$ & slow & $S O M$ & -39.43 & 112.41 & 3.24 & 3.41 & 5.59 \\
\hline$P B W$ & fast & $S O M$ & -56 & 170.67 & 3.24 & 7.95 & 4.69 \\
\hline$S O M$ & slow & $P B W$ & -64.73 & 25.62 & 6.92 & 1.86 & 1.33 \\
\hline SOM & fast & $P B W$ & -58.57 & 23.5 & 6.92 & 1.91 & 2.39 \\
\hline SOM & slow & $S L U$ & -73.99 & 27.73 & 6.92 & 1.63 & 0.45 \\
\hline SOM & fast & $S L U$ & -53.41 & 20.96 & 6.92 & 1.88 & 2.74 \\
\hline SOM & slow & LWR & -70.68 & 116.63 & 3.64 & -8.6 & 5.3 \\
\hline SOM & fast & LWR & -38.29 & 150.28 & 3.64 & 24.21 & 10.22 \\
\hline SOM & slow & $S O M$ & -41.07 & 62.01 & 4.52 & 4.02 & 5.22 \\
\hline$S O M$ & fast & $S O M$ & -21.28 & 102.47 & 4.52 & 32.85 & 31.40 \\
\hline
\end{tabular}

PBW Paperback Writer; SOM Something; SLU She Loves You; LWR The Long and Winding Road; $S_{M I N}$ minimum subjective value from rangefrequency fit; $S_{M A X}$ maximum subjective value from range-frequency fit; $J_{I D E A L}$ judgment value corresponding to the ideal in Eq. 2 . Predicted ideal $=\mathrm{S}_{\mathrm{MIN}}+\left(\mathrm{J}_{\text {Ideal }}{ }^{-1}\right)\left(\mathrm{S}_{\mathrm{MAX}}-\mathrm{S}_{\mathrm{MIN}}\right) / 8$; Inferred ideal from the fit of Eq. 4

and $S L U$, which was rated as similar to $P B W$, but did not affect tempo ratings of $S O M$ and $L W R$, which were rated as dissimilar to $P B W$. Likewise, manipulation of the $S O M$ context affected tempo ratings of $S O M$ and $L W R$, which was rated as similar to $S O M$, but did not affect tempo ratings of $P B W$ and $S L U$, which were rated dissimilar to $S O M$. Although the contextual manipulation of tempo did not significantly alter tempo ratings for dissimilar songs, an examination of Fig. 9 indicates that the dissimilar songs still had an impact on tempo judgment. For example, the lowest rating for $P B W$ in the $P B W$ slow context was 3.42 in Experiment 3 but was 2.12 in Experiment 2 (compare with Fig. 6). The key difference between these conditions was that in Experiment 3, we included the slow tempo target songs $S O M$ and $L W R$. Thus, it would seem that including these dissimilar songs affected tempo judgments. Similarly, the highest tempo rating for SOM shown in Fig. 9 is only 5.22, which would likely have been much higher if the faster tempo target songs $P B W$ and $S L U$ had not been included. Why, then, were target song tempo ratings not significantly affected by the manipulation of the contextual set for the dissimilar context song? It may be that ratings of tempo were primarily based on rank within context rather than range shifts. The dissimilar songs were very different in tempo so that both fast and slow tempo contexts did not change the ranks of the target tempos (see Table 1) and hence would not produce a context effect based on the frequency principle alone (Parducci, 1995).
Regardless of the interpretation of the lack of context effects on dissimilar songs, a key test of Experiment 3 was to determine whether ideal point shifts for pleasantness ratings would follow the same contextual pattern as contrast effects on tempo ratings. If so, it would be consistent with a judgment-mediated model, according to which the ideal shifts because of shifts in the perceived tempo. However, if the prototype model is correct, then the pleasantness shift should be based on the distribution of tempos for the same song in memory, and therefore one would expect these effects to be song specific. The results of Experiment 3 provide strong support for song-specific shifts in ideals, consistent with the prototype model and inconsistent with the judgment-mediated model. This result was particularly convincing for the $P B W$ context, in which none of the other targets showed context effects on pleasantness ratings. Also consistent with this result, the only significant shift in ideal points for the SOM context was when the target matched the context. Furthermore, the predictions of the judgmentmediated model generated from tempo judgment effects shown in Table 4 confirm that the model incorrectly predicts shifts of ideal points on similar songs that did not occur. Although ideal points did not shift with context for similar songs, there was some additional indication of context effects on pleasantness judgments of the similar target $L W R$. It seems that when the context is strongly pleasant or unpleasant, it may lead to displacement of targets in the opposite direction on the pleasantness scale, a hedonic 
contrast effect that has been reported previously in the literature (Parker et al., 2008). Thus, it appears that multiple contextual effects may be operating on pleasantness ratings.

\section{General discussion}

In Experiments 1-3, we demonstrated large and rapidly developing context effects for Beatles music samples on both tempo and pleasantness judgments. Note that there was no preview of the contextual distribution of songs, and yet strong context effects were found in the first set of judgments, which consisted of just five target and seven context samples. Furthermore, when the context was shifted to the opposite condition after the first full block of ratings, judgments shifted significantly as well. Finally, the robustness of these effects was demonstrated across different levels of participants' familiarity with the songs. Thus, it would appear that listening to an extreme tempo for a song quickly changes how other tempo versions of the song are judged.

The combined results of the three experiments provide further evidence that context effects on attribute judgments can be dissociated from context effects on ideal points derived from pleasantness judgments. In both Experiments 1 and 2, within-subjects variation in contrast effects across contexts did not correlate with variation in the most pleasant target tempos across contexts. These results were also found in regression analyses that attempted to control for differences in slopes of the tempo rating functions between participants and between contexts. The lack of correlation for within-subjects manipulations of context on tempo and pleasantness ratings is consistent with a similar lack of correlation reported in other studies (Pettibone \& Wedell, 2007) and on face value argues against the judgmentmediated model, which predicts the two effects should be closely linked. However, analyses of the judgment-mediated model predictions shown in Table 3 indicate that the model does well in predicting context effects on tempo pleasantness for the first context encountered but not for the second context encountered. One interpretation of this result is that the judgment mediation may drive the effects initially, but that additional processes come into play when the context is shifted a second time. According to this view, carryover effects are different for attribute judgment than for attribute pleasantness, perhaps with the latter being more resistant to change.

However, results of Experiment 3 indicate that the poor fit of the judgment-mediated model is not restricted to the case of manipulating context within subject. In Experiment 3 , context was manipulated between subjects, and the effects of the manipulation on different songs were evaluated. Inconsistent with the judgment-mediated model, contrast effects transferred to similar songs, but ideal-point shifts did not. This result demonstrates a clear dissociation between context effects on tempo and pleasantness that is inconsistent with a one-process model of these effects. Instead, the results of Experiment 3 are consistent with a prototype model of pleasantness in which the ideal values shift toward the mean of recently experienced values (Wedell \& Pettibone, 1999). The song-specific nature of contextual recruitment for pleasantness judgments found in Experiment 3 is what one would expect if the target song was used as a memory probe to recruit instances in memory from which to construct a prototype (Hintzman, 1986). The prototype model is also consistent with results in the attractiveness literature in which the average of stimuli is often judged more attractive than the constituents, both for faces (Langlois \& Roggman, 1990) and for musical performances (Repp, 1997).

The song-specific nature of the shifts in ideal points observed in Experiment 3 would appear to limit the degree to which contextual shifts in tempo will be observed in realworld settings. For example, in a concert setting, the same song is rarely played twice. If results of Experiment 3 are taken at face value, then listening to a slowly played first song of a concert will not generally shift the preferred tempo for the second song to a slower tempo. Of course, what constitutes the "same" is not always easy to determine. It may be that structurally similar songs are recruited in making evaluations, so a highly similar song within a concert may alter the preferred tempo of a target song. If so, the songs used in Experiment 3 may simply not have been similar enough to be included in the same context-determining ideals, but other more similar songs may have done so.

The pervasive context effects demonstrated in Experiments 1-3 may seem at odds with the idea promoted by Levitin and Cook (1996) that absolute tempo is preserved in memory. However, it should be noted that their research did not attempt to measure the contextual sensitivity of reproduced tempos, but merely demonstrated that very familiar songs were reproduced at fairly accurate tempo rates (+/8\%). McAuley and colleagues (Jones \& McAuley, 2005; McAuley \& Miller, 2007) demonstrated how perceived tempo might shift with local context (sequence effects) and global context (distribution effects), so there are clear contradictions to the hypothesis of absolute tempo memory. On the other hand, note that the equations we used to describe contextual effects on tempo generally assume that absolute values of tempo $\left(\mathrm{S}_{i}\right)$ are acted upon, but these absolute values may be used in a relative way when judging the tempo value. Indeed, the nature of the context effects observed may depend strongly on the nature of the task in which the participant is engaged.

Throughout this article, we have referred to the ideal as the most pleasant tempo rather than using the term the most preferred tempo. This is because in the tempo literature, the term preferred tempo has been used to refer to the tempo 
that optimizes perceptual performance (Fraisse, 1963; Moelants, 2002). Jones and McAuley (2005) demonstrated that the tempo that minimized absolute error and bias corresponded to the mean of the distribution of tempos presented in an experiment. This result implied that the optimal or preferred tempo is not absolute as suggested by Fraisse, but is context dependent. Our focus on pleasantness judgments reflects a focus on preferred tempo in an aesthetic sense, rather than in the optimizing sense. It is interesting that the preferred tempo in the aesthetic sense, as measured in the present set of experiments, tends to shift toward the preferred tempo in the optimizing sense, which corresponds to the mean of the recent set of tempos. Future research should examine the correspondence of these two types of tempo preference.

Although judgment-mediated pleasantness shifts were not demonstrated in the present set of experiments, this does not mean that rank- and range-based preference reversals are not pervasive. When multiple dimensions are used to describe stimuli, range-frequency effects on the underlying dimensions predict preference reversals when these dimensions are combined in judgment and choice. Such predictions have been borne out by a large body of research (Cooke \& Mellers, 1998; Mellers \& Cooke, 1996; Stewart, Chater, \& Brown, 2006; Ungemach, Stewart \& Reimers, 2011; Wedell, 1998). However, research on shifts of ideals along a single dimension appears to support a separate process based on averages rather than ranks (Pettibone \& Wedell, 2007; Wedell et al., 2005). Thus, there are at least two distinct contextual processes that may lead to context dependent preference reversals.

Finally, in speculating about the implications of this work, we believe that altered tempo versions of music may become widespread in the future. Since most music is processed by computers these days, the ability to change tempo of music without changing pitch may become another factor, such as treble or bass on one's stereo system. If simple controls to adjust tempo become ubiquitous in the future in sound systems, then it is likely that people will expose themselves to tempo-altered versions of songs they know. Our research implies that exposure to song-specific tempoaltered versions of familiar music will immediately affect the song's tempo pleasantness in listeners regardless of familiarity. However, any long-term effects of exposure to tempo-altered versions are unknown in listeners.

In the area of music composition, the effects of exposure to tempo-altered versions may cause composers to choose faster or slower tempos in their original pieces. Since many composers currently write their music using a computer, and software enables them to preview and change the tempo of their compositions, they will likely try out their pieces at different speeds and then decide on the most pleasant tempo. For example, if composers expose themselves to faster versions of the piece they are working on, they will likely set the tempo at a faster value. However, because performers of these pieces have not been exposed to the same context as the composer, they may feel as though the tempo is not pleasant. Indeed, composers may also feel the tempo is not pleasant during performance if their last exposure to the extreme context occurred days or weeks beforehand. In a digital age in which attributes of a song may be freely manipulated by the listener, composer, and performer, we expect to find widespread examples of the contextual dependence of pleasantness in music aesthetics.

\section{References}

Ariely, D. (2001). Seeing sets: Representation by statistical properties. Psychological Science, 12, 157-162.

Brown, D. R. (1953). Stimulus similarity and the anchoring of subjective scales. The American Journal of Psychology, 66, 199-214.

Cooke, A. D. J., Janiszewski, C., Cunha, M., Jr., Nasco, S. A., \& de Wilde, E. (2004). Stimulus context and the formation of consumer ideals. Journal of Consumer Research, 31, 112-124.

Cooke, A. D. J., \& Mellers, B. A. (1998). Multiattribute judgments: Context effects in single attributes. Journal of Experimental Psychology: Human Perception and Performance, 24, 496-504.

Coombs, C. H. (1964). A theory of data. New York, NY: Wiley.

Deutsch, D. (1999). The psychology of music (2nd ed.). New York, NY: Academic Press.

Eagly, A., \& Chaiken, S. (1993). The psychology of attitudes. Orlando, FL: Harcourt Brace Jovanovich.

Edworthy, J., \& Waring, H. (2006). The effects of music tempo and loudness level on treadmill exercise. Ergonomics, 49, 1597-1610.

Fraisse, P. (1963). The psychology of time. New York, NY: Harper \& Row.

Geringer, J. M. (2010). Musicians' preference for tempo and pitch levels in recorded orchestra music. Journal of Research in Music Education, 58, 294-308.

Helson, H. H. (1964). Adaptation-level theory. New York, NY: Harper and Row.

Hicklin, S. K., \& Wedell, D. H. (2007). Learning group differences: Implications for contrast and assimilation in stereotyping. Social Cognition, 25, 410-454.

Hintzman, D. L. (1986). "Schema abstraction" in a multiple-trace memory model. Psychological Review, 93, 411-428.

Holbrook, M. B., \& Anand, P. (1990). Effects of tempo and situational arousal on the listener's perceptual and affect response to music. Psychology of Music, 18, 150-162.

Homa, D. (1984). On the nature of categories. Psychology of Learning and Motivation, 18, 49-94.

Honing, H. (2006). Evidence for tempo-specific timing in music using a web-based experimental setup. Journal of Experimental Psychology: Human Perception and Performance, 32, 780-786.

Honing, H., \& Ladinig, O. (2009). Exposure influences expressive timing judgments in music. Journal of Experimental Psychology: Human Perception and Performance, 35, 281-288.

Jones, M. R., \& McAuley, J. D. (2005). Time judgments in global temporal contexts. Perception \& Psychophysics, 67, 398-417.

Langlois, J. H., \& Roggman, L. A. (1990). Attractive faces are only average. Psychological Science, 1, 115-121.

LeBlanc, A., Colman, J., McCrary, J., Sherrill, C., \& Malin, S. (1988). Tempo preferences of different age music listeners. Journal of Research in Music Education, 36, 156-168. 
LeBlanc, A., \& McCrary, J. (1983). Effect of tempo on children's music preference. Journal of Research in Music Education, 31, 283-294.

Leonard, H. (1994). The Beatles 1962-1966 (guitar recorded version). Milwaukee, Wisconsin; Hal Leonard Corporation.

Leonard, H. (1995). The Beatles 1967-1970 (guitar recorded version). Hal Leonard Corporation.

Levitin, D. J., \& Cook, P. R. (1996). Memory for musical tempo: Additional evidence that auditory memory is absolute. Perception \& Psychophysics, 58, 927-935.

Lockhead, G. R., \& King, M. C. (1983). A memory model of sequential effects in scaling tasks. Journal of Experimental Psychology: Human Perception and Performance, 9, 461-473.

McAuley, J. D., \& Miller, N. S. (2007). Picking up the pace: Effects of global temporal context on sensitivity to the tempo of auditory sequences. Perception \& Psychophysics, 69, 708-718.

Mellers, B. A., \& Cooke, A. D. J. (1996). The role of task and context in preference measurement. Psychological Science, 7 , $76-82$.

Moelants, D. (2002). Preferred tempo reconsidered. In C. Stevens, D. Burnham, G. McPherson, E. Schubert, \& J. Renwick (Eds.), Proceedings of the 7th International Conference on Music Perception and Cognition (pp. 580-583). Adelaide, South Australia, Australia: Causal Productions.

Nosofsky, R. M., \& Zaki, S. R. (2002). Exemplar and prototype models revisited: Response strategies, selective attention, and stimulus generalization. Journal of Experimental Psychology: Learning, Memory, and Cognition, 28, 924-940.

O'Reilly, D. M., Leitch, R. A., \& Wedell, D. H. (2004). The effects of immediate context on auditors judgments of loan quality. Auditing: A Journal of Practice and Theory, 23, 89-105.

Parducci, A. (1956). Direction of shift in the judgment of single stimuli. Journal of Experimental Psychology, 51, 169-178.

Parducci, A. (1995). Happiness, pleasure, and judgment: The contextual theory and its applications. Mahwah, New Jersey: Erlbaum.

Parducci, A., Knoble, S., \& Thomas, C. (1976). Independent contexts for category ratings: A range-frequency analysis. Perception \& Psychophysics, 20, 360-366.

Parker, S., Bascom, J., Rabinovitz, B., \& Zellner, D. (2008). Positive and negative hedonic contrast with music stimuli. Psychology of Aesthetics, Creativity, and the Arts, 2, 171-174.

Perrett, L. F. (1971). Immediate and background contextual effects in clinical judgment. Unpublished doctoral dissertation, University of California, Los Angeles.
Pettibone, J. C., \& Wedell, D. H. (2007). Of gnomes and leprechauns: The recruitment of recent and categorical contexts in social judgment. Acta Psychologica, 125, 361-389.

Quin, S., \& Watt, R. (2006). The perception of tempo in music. Perception, 35, 267-280.

Reed, S. K. (1972). Pattern recognition and categorization. Cognitive Psychology, 3, 383-407.

Repp, H. B. (1997). The aesthetic quality of a quantitatively average music performance: Two preliminary experiments. Music Perception, 14, 419-444.

Riskey, D. R., Parducci, A., \& Beauchamp, G. K. (1979). Effects of context in judgments of sweetness and pleasantness. Perception \& Psychophysics, 26, 171-176.

Rota, L. M., \& Zellner, D. A. (2007). The categorization effect in hedonic contrast: Experts differ from novices. Psychonomic Bulletin \& Review, 14, 179-183.

Sarris, V. (1976). Effects of stimulus range and anchor value on psychophysical judgment. In H.-G. Geissler \& Y. M. Zaprodin (Eds.), Advances in psychophysics (pp. 253-268). Berlin, Germany: VEB Deutscher Verlag der Wissenschaften.

Stewart, N., Chater, N., \& Brown, G. D. A. (2006). Decision by sampling. Cognitive Psychology, 53, 1-26.

Ungemach, C., Stewart, N., \& Reimers, S. (2011). How incidental values from our environment affect decisions about money, risk, and delay. Psychological Science, 22, 523-560.

Waterhouse, J., Hudson, P., \& Edwards, B. (2010). Effects of music tempo upon submaximal cycling performance. Scandinavian Journal of Medicine \& Science in Sports, 20, 662-669.

Wedell, D. H. (1998). Testing models of tradeoff contrast in pairwise choice. Journal of Experimental Psychology: Human Perception and Performance, 24, 49-65.

Wedell, D. H., Hicklin, S. K., \& Smarandescu, L. O. (2007). Contrasting models of assimilation and contrast. In D. A. Stapel \& J. Suls (Eds.), Assimilation and contrast in social psychology (pp. 4574). New York, NY: Psychology Press.

Wedell, D. H., \& Pettibone, J. C. (1999). Preference and the contextual basis of ideals in judgment and choice. Journal of Experimental Psychology: General, 128, 346-361.

Wedell, D. H., Santoyo, E. M., \& Pettibone, J. C. (2005). The thick and the thin of it: Contextual effects in body perception. Basic and Applied Social Psychology, 27, 213-227.

Zellner, D. A., Rohm, E. A., Bassetti, T. L., \& Parker, S. (2003). Compared to what? Effects of categorization on hedonic contrast. Psychonomic Bulletin \& Review, 10, 468-473. 